\title{
Selection of an instrument to evaluate the organizational environment of nurses working in intensive care: an integrative review
}

\author{
Brett Abbenbroek, Christine Duffield, Doug Elliott \\ Centre for Health Services Management, Faculty of Health, University of Technology, Sydney, Australia \\ Correspondence: Brett Abbenbroek. Address: Centre for Health Services Management, Faculty of Health, University of \\ Technology, Sydney, 15 Broadway Ultimo, NSW 2007, Australia. E-mail: Brett.J.Abbenbroek@student.uts.edu.au \\ Received: August 18, 2014 \\ DOI : $10.5430 /$ jha.v3n6p143 \\ Accepted: October 16, 2014 \\ Online Published: October 31, 2014 \\ URL: http://dx.doi.org/10.5430/jha.v3n6p143
}

\begin{abstract}
Objective: To determine an appropriate survey instrument to evaluate the impact of organizational structures on the work environment of intensive care nurses.

Background: Internationally the demand for intensive care is increasing. Solely increasing bed capacity is not sustainable. Large capacity multi-specialty Intensive Care Units are emerging as the preferred organizational model with benefits resulting from optimizing operational synergies and economies of scale. The impact of this organizational transition on intensive care nurses is not well understood. An appropriate survey instrument for intensive care nurses is required. Design: Integrative literature review. Data Sources: CINAHL, PubMed, EMBASE and OVID Nursing databases searched for studies published between 2005 and 2013.

Review methods: An integrative review and quality assessment of the studies was undertaken to select nurse outcome measures associated with organizational structures across a range of acute and critical care settings. Congruence between nurse outcome measures and nurse survey instruments tested in the literature was assessed to select instruments for further psychometric evaluation.
\end{abstract}

Results: Thirty-one cross sectional quantitative studies, from fourteen countries, were reviewed. Twenty one nurse outcome measures associated with organizational factors were identified and a total of twenty five survey instruments used in the studies reviewed. Assessment of congruence and psychometric properties determined that a combination of two instruments is required to comprehensively assess the organizational environment of nurses working in intensive care units.

Conclusion: The environment of nurses working in intensive care is effectively evaluated with an instrument that combines subscales from the Practice Environment Scale-Nurse Work Index and Maslach's Burnout Inventory.

\section{Key words}

Nurse, Intensive care, Critical care, Organization, Environment, Outcome, Satisfaction 


\section{Introduction}

Intensive Care Units (ICUs) support critically ill patients that require complex clinical management, sophisticated technologies and high resource inputs. Internationally, the demand for intensive care is growing due to aging populations, higher inpatient acuity with increasing multiple co-morbidities and advanced medical technologies ${ }^{[1,2]}$.

Effective demand management aims to improve utilization of available bed capacity while optimizing patient and staff outcomes ${ }^{[3]}$. An established demand management strategy is coordinated networking between hospitals for the referral of critically ill patients to access definitive care ${ }^{[4,5]}$. As a result organizational transformation in the form of regionalization, or consolidation, of ICU services is being adopted across clinical networks and within individual hospitals ${ }^{[6]}$.

Large-capacity multi-specialty ICUs are emerging as the preferred organizational model in tertiary and regional referral hospitals where historically multiple sub-specialty ICUs operated separately ${ }^{[2,4,7]}$. Typically these units range from fifty to seventy beds, in contrast to the traditional ICU model of between ten and twelve beds, and require a large clinical workforce ${ }^{[7-9]}$.

Benefits are thought to be linked to consolidation and better utilization of expertise and resources ${ }^{[2,10]}$. Flexible patient flow, economies of scale, enhanced operational synergies and standardization of practice underpin the benefits achieved $^{[11-13]}$.

Increasing bed capacity alone is not sustainable, however, in terms of both fiscal and human resources ${ }^{[14,15]}$. Structural changes to the work environment are required to achieve organizational transformation and include nursing management models, nurse staffing, rostering, professional development and the need for a large nursing workforce ${ }^{[13]}$.

A major challenge is effective management of the large nurse workforce required on a 24-hour basis, so as to optimize nurse outcomes such as staff satisfaction and retention ${ }^{[16,17]}$. Nurse outcomes have been investigated in acute care environments ${ }^{[18,19]}$, however, intensive care nurse outcomes are not so well understood and may result in the adoption of unsustainable organizational models ${ }^{[20-22]}$. A survey instrument sensitive to organizational factors and culture, with strong psychometric properties, is required to evaluate the working environment of intensive care nurses, inform managers and promote workforce sustainability in the face of organizational change.

\section{Method}

An integrative literature review of the empirical literature was conducted using methodological approaches described by Cooper (1982) ${ }^{[23]}$ and Dixon-Woods et al. (2004) ${ }^{[24]}$ for integrative reviews of quantitative and qualitative research. An integrative approach includes a diverse range of study designs, if present in the literature, thereby providing a broad perspective that enriches the understanding of the topic ${ }^{[25]}$. Key review stages included a review of acute care nurse outcome studies, quality assessment, identifying nurse outcome measures and the survey instruments tested, followed by an assessment of the selected instrument psychometric properties.

\subsection{Search method}

During the literature search stage, the first author interrogated the CINAHL, PubMed, EMBASE and OVID Nursing databases for English language studies published internationally between 2005 and 2013 (December). Early literature from 2005 was included to capture seminal studies by Manojlovich et al. (2005) ${ }^{[26]}$ and Stone et al. (2006) ${ }^{[3]}$. The keyword used for the search was "nurse" with advanced searching cross-referencing the search terms "intensive care", "critical care", "ICU”, "environment", "organization", “outcome" and "satisfaction".

Studies reviewed were included based on the following criteria: (1) empirical study reports; (2) studies conducted in an acute care environment; (3) explicit study of the association between nurse outcomes and organizational factors; and 
(4) psychometric properties of the survey instrument used was defined. Exclusion criteria included: (1) non-English language publications; (2) non-adults; and (3) editorials or unpublished dissertations.

\subsection{Search results}

The search yielded a total of 309 studies of which thirty nine were initially retained. Further analysis excluded three studies that used either a locally developed non-validated survey instrument tested on a small sample of nurses ${ }^{[27,28]}$ or focused on nurse-sensitive patient outcomes ${ }^{[29]}$. Five studies were excluded as they focused solely on validating survey instruments through subscale factor analysis ${ }^{[31-34]}$. The procedure and outcomes are outlined in Figure 1.

Figure 1. Literature search summary flow chart

Note. Definitions of survey instruments available from the author on request.

\begin{tabular}{|c|c|}
\hline $\begin{array}{l}\text { I references identified through datassse searches } \\
\qquad(n=312)\end{array}$ & \multirow{7}{*}{ 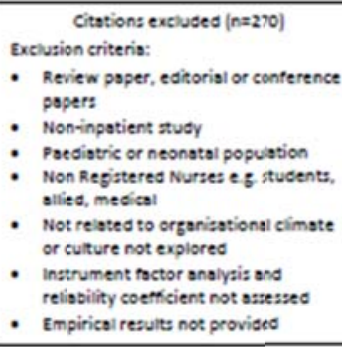 } \\
\hline 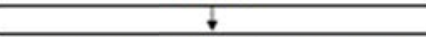 & \\
\hline $\begin{array}{l}\text { = sdcitional references identified from refterence } \\
\qquad l i s t s(n=14)\end{array}$ & \\
\hline 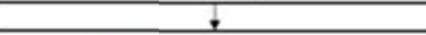 & \\
\hline $\begin{array}{l}\text { = duplicate reterences removed } \\
\qquad(n=17)\end{array}$ & \\
\hline+ & \\
\hline $\begin{array}{l}\text { a reterences screened } \\
\qquad[n=309]\end{array}$ & \\
\hline 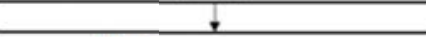 & \\
\hline = full teit references assessed & Citations excluced $(n=1)$ \\
\hline (n= 39) & - Survey instrument developnent \\
\hline$\frac{1}{t}$ & 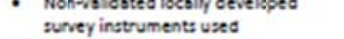 \\
\hline $\begin{array}{l}=\text { studies included in qualitative synthesis } \\
\qquad(n=31)\end{array}$ & $\begin{array}{l}\text { - Non-Englith language } \\
\text { - Primanily patient outcomes tudied }\end{array}$ \\
\hline$F$ & 7 \\
\hline 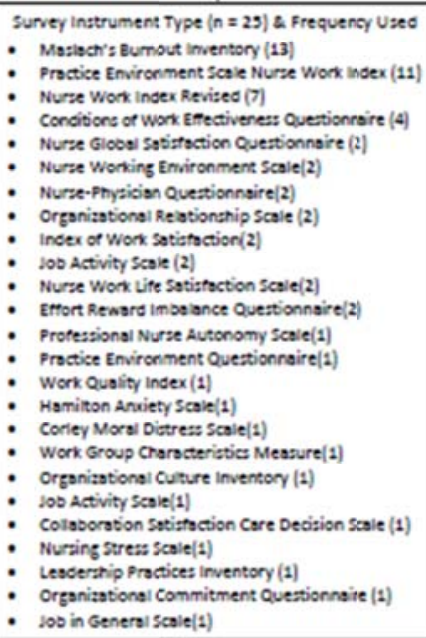 & 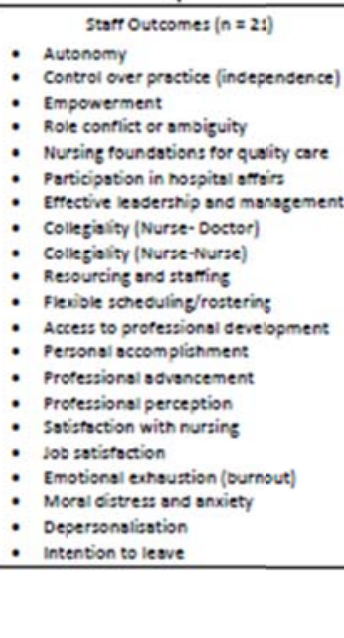 \\
\hline
\end{tabular}

\section{Conclusion}

Thirty one studies were retained for full analysis as summarized in Table 1. Twenty one nurse outcomes were identified for further exploration. Twenty five survey instruments were used either singularly or in combination as listed in Figure 1. A comprehensive quality appraisal was then undertaken to further validate the inclusion of identified studies in this review.

\subsection{Quality appraisal}

Quality was assessed based on criteria recommended in the Critical Review of Quantitative Research Worksheet and aligned with the methods promoted in the Strengthening the Reporting of Observational Studies in Epidemiology (STROBE) statement ${ }^{[35,36]}$. Each criteria was assessed using an allocated score based on the evidence hierarchy proposed by Evans $(2003)^{[37]}$. This scoring process, first developed by Beck $(1995)^{[38]}$ and applied recently to a literature review of nurse turnover costs conducted by Li et al. (2012) ${ }^{[39]}$, was adopted and expanded with additional quality criteria proposed by Miller (2006) ${ }^{[35]}$. 
Table 1. Literature review result summary

\begin{tabular}{|c|c|c|c|c|c|c|c|c|c|c|}
\hline \multirow[b]{2}{*}{ Author } & \multirow{2}{*}{$\begin{array}{c}\text { Study } \\
\text { Design }\end{array}$} & \multirow{2}{*}{$\begin{array}{c}\text { Survey } \\
\text { Tool }\end{array}$} & \multirow{2}{*}{$\begin{array}{l}\text { Cronbach } \alpha \\
\text { (composite) }\end{array}$} & \multirow{2}{*}{$\begin{array}{c}\text { Sample } \\
\text { (n) }\end{array}$} & \multirow{2}{*}{$\begin{array}{c}\text { Inpatient } \\
\text { Clinical } \\
\text { Unit }\end{array}$} & \multicolumn{5}{|c|}{ Workplace Evaluation Results } \\
\hline & & & & & & Outcome & Variable & Stat $^{ \pm}$ & $\begin{array}{c}95 \% \\
\text { CI/SD }\end{array}$ & $*$ \\
\hline \multirow{25}{*}{$\begin{array}{l}\text { Van } \\
\text { Bogaert } \\
\text { et al. } \\
2013 \text {, } \\
\text { Belgium }\end{array}$} & \multirow{25}{*}{$\begin{array}{l}\mathrm{PCS}^{\dagger} \\
\text { Survey }\end{array}$} & \multirow{25}{*}{$\begin{array}{l}\text { NWI-R } \\
\text { MBI }\end{array}$} & & & & Job satisfaction & $\begin{array}{l}\text { Nurse-Physician } \\
\text { collegiality }\end{array}$ & OR 2.28 & $1.46-3.54$ & $<0.001$ \\
\hline & & & & & & & Nurse management (unit) & OR 10.7 & $4.97-23.06$ & $<0.001$ \\
\hline & & & & & & & Organisational support & OR 9.42 & $4.23-20.96$ & $<0.001$ \\
\hline & & & & & & & Workload & OR 0.35 & $0.21-0.57$ & $<0.001$ \\
\hline & & & & & & & Autonomy & OR 5.27 & $2.45-11.36$ & $<0.001$ \\
\hline & & & & & & & Emotional exhaustion & OR 0.40 & $0.33-0.49$ & $<0.001$ \\
\hline & & & & & & & Depersonalisation & OR 0.55 & $0.44-0.68$ & $<0.001$ \\
\hline & & & & & & & Personal & OR 1.62 & $1.25-2.12$ & $<0.001$ \\
\hline & & & & & & & accomplishment & OR 1.71 & $1.13-2.59$ & $<0.05$ \\
\hline & & & & & & $\begin{array}{l}\text { No intention to } \\
\text { leave }\end{array}$ & Nurse-Physician & OR 410 & & \\
\hline & & & & & $\begin{array}{l}\text { Wide } \\
\text { acute care }\end{array}$ & & $\begin{array}{l}\text { collegiality } \\
\text { Nurse management (unit) }\end{array}$ & $\begin{array}{l}\text { OR } 4.10 \\
\text { OR } 9.42\end{array}$ & $\begin{array}{l}2.05-8.21 \\
2.09-8.53\end{array}$ & $\begin{array}{l}<0.001 \\
<0.001\end{array}$ \\
\hline & & & & & $(\mathrm{n}=8$ & & Organisational support & OR 0.56 & $0.36-0.87$ & $\mathrm{n} / \mathrm{s}^{5}$ \\
\hline & & & 0.80 & $1108 \mathrm{RN}$ & hospitals, & & Workload & OR 1.82 & $0.93-3.57$ & $\mathrm{n} / \mathrm{s}$ \\
\hline & & & & & nursing & & Autonomy & OR 0.59 & $0.49-0.71$ & $<0.001$ \\
\hline & & & & & units & & Emotional exhaustion & OR 0.72 & $0.58-0.89$ & $<0.001$ \\
\hline & & & & & & & Depersonalisation & OR 1.64 & $1.28-2.12$ & $<0.01$ \\
\hline & & & & & & & $\begin{array}{l}\text { Personal } \\
\text { accomplishment }\end{array}$ & OR 2.92 & $1.89-4.51$ & $<0.001$ \\
\hline & & & & & & Quality of care & Nurse-Physician & & & \\
\hline & & & & & & (perceived) & collegiality & OR 50.2 & $19.67-128$ & $<0.001$ \\
\hline & & & & & & & Nurse management (unit) & OR 6.87 & $3.52-14.25$ & $<0.001$ \\
\hline & & & & & & & Organisational support & OR 0.77 & $0.49-1.20$ & $\mathrm{n} / \mathrm{s}$ \\
\hline & & & & & & & Workload & OR 7.03 & $3.36-14.71$ & $<0.001$ \\
\hline & & & & & & & Autonomy & OR 0.68 & $0.57-0.82$ & $<0.001$ \\
\hline & & & & & & & Emotional exhaustion & OR 0.66 & $0.53-0.82$ & $<0.001$ \\
\hline & & & & & & & Depersonalisation & OR 1.48 & $1.16-1.88$ & $<0.001$ \\
\hline & & & & & & Job satisfaction & $\begin{array}{l}\text { Perceived leadership } \\
\text { qualities: }\end{array}$ & & & \\
\hline & & & & & & & Modelling the way & $r=0.23$ & $n / a^{4}$ & $=0.01$ \\
\hline $\begin{array}{l}\text { Moneke } \\
\text { et } a l\end{array}$ & & & 0.95 & & & & Inspiring shared vision & $r=0.24$ & $\mathrm{n} / \mathrm{a}$ & $=0.01$ \\
\hline 2013 & Survey & OCM & 0.86 & $112 \mathrm{RN}$ & ICU & & Challenging the process & $r=0.23$ & $\mathrm{n} / \mathrm{a}$ & $=0.02$ \\
\hline & & & 0.87 & & & & Enabling & $r=0.21$ & $\mathrm{n} / \mathrm{a}$ & $=0.02$ \\
\hline & & & & & & & Ecouraging the heart & $r=0.13$ & $\mathrm{n} / \mathrm{a}$ & $=0.15$ \\
\hline & & & & & & & $\begin{array}{l}\text { Organisational } \\
\text { commitment }\end{array}$ & $\beta=0.35$ & $\mathrm{n} / \mathrm{a}$ & $=0.00$ \\
\hline & & & & & & Job satisfaction & Emotional exhaustion & $r=-0.41$ & $\mathrm{n} / \mathrm{a}$ & $<0.001$ \\
\hline & & & & & & & Depersonalisation & $r=-0.31$ & $\mathrm{n} / \mathrm{a}$ & $<0.001$ \\
\hline Myhren & & & & & & Job stress & Personal & & & \\
\hline et al. & & MBI & 070 & $129 \mathrm{RN}$ & ICUs & & accomplishment & $r=0.12$ & $\mathrm{n} / \mathrm{a}$ & $=0.145$ \\
\hline 2013, & PDC & MBI & 0.10 & $129 \mathrm{RN}$ & $(\mathrm{n}=3)$ & & Emotional Exhaustion & $r=0.586$ & $\mathrm{n} / \mathrm{a}$ & $<0.001$ \\
\hline & & & & & & & Depersonalisation & $r=0.293$ & $\mathrm{n} / \mathrm{a}$ & $<0.001$ \\
\hline & & & & & & & $\begin{array}{l}\text { Personal } \\
\text { accomplishment }\end{array}$ & $r=0.105$ & $\mathrm{n} / \mathrm{a}$ & $=0.208$ \\
\hline & & & & & & Burnout & Practice Environment & OR 0.55 & $0.41-0.75$ & $=0.01$ \\
\hline & & & & & & & Workload & OR 1.03 & $0.96-1.10$ & $\mathrm{n} / \mathrm{s}$ \\
\hline & & & & & & Job & Practice Environment & OR 0.47 & $0.34-0.66$ & $=0.01$ \\
\hline & & & & & & dissatisfaction & Workload & OR 1.04 & $0.96-1.13$ & $\mathrm{n} / \mathrm{s}$ \\
\hline & & & & & Hospital & & & & & \\
\hline et al. & & & & & wide & Intent to leave & Practice Environment & OR 0.64 & $0.49-0.84$ & $=0.01$ \\
\hline 2013, & $\mathrm{PCS}$ & PESWI & 0.79 & $1187 \mathrm{RN}$ & acute & & Workload & OR 1.04 & $0.99-1.10$ & $\mathrm{n} / \mathrm{s}$ \\
\hline South & Survey & MBI & 0.88 & & care, $(\mathrm{n}=$ & Poor quality & Practice Environment & OR 0.55 & $0.41-0.74$ & $=0.01$ \\
\hline Africa & & & & & & & Workload & OR 1.06 & $1.01-1.12$ & $=0.05$ \\
\hline & & & & & hospitals) & Poor & Practice Environment & OR 0.41 & $0.31-0.55$ & $=0.01$ \\
\hline & & & & & & Management & Workload & OR 1.01 & $0.92-1.12$ & $\mathrm{n} / \mathrm{s}$ \\
\hline & & & & & & Poor safety & Practice Environment & OR 0.48 & $0.29-1.02$ & $\mathrm{n} / \mathrm{s}$ \\
\hline & & & & & & & Workload & OR 1.11 & $1.00-1.24$ & $=0.05$ \\
\hline & & & & $\mathrm{EU}$ & & (EU) & & & & \\
\hline & & & & 33,659 & & Poor quality & Practice Environment & OR 0.56 & $0.51-0.61$ & $<0.05$ \\
\hline & & & & & & Poor safety & Practice Environment & OR 0.50 & $0.44-0.56$ & $<0.05$ \\
\hline & & & & & & Burnout & Practice Environment & OR 0.67 & $0.61-0.73$ & $<0.05$ \\
\hline & & & & & Hospital & Dissatisfaction & Practice Environment & OR 0.52 & $0.47-0.57$ & $<0.05$ \\
\hline & & & & & wide & Intent to Leave & Practice Environment & OR 0.61 & $0.56-0.67$ & $<0.05$ \\
\hline al. 2012 , & $\mathrm{PCS}^{\dagger}$ & PES-N & $\begin{array}{l}\text { Referenced } \\
\text { from other }\end{array}$ & & $\begin{array}{l}\text { acute care } \\
(\mathrm{n}=\end{array}$ & Poor mgmt. & Practice Environment & OR 0.53 & $0.48-0.58$ & $<0.05$ \\
\hline Europe & Survey & WI MBI & studies & 27,509 & $488 \mathrm{EU}+$ & (US) & & & & \\
\hline$\&$ US & & & & & 612US & Poor quality & Practice Environment & OR 0.54 & $0.51-0.58$ & $<0.05$ \\
\hline & & & & & & Poor safety & Practice Environment & OR 0.55 & $0.50-0.61$ & $<0.05$ \\
\hline & & & & & & Burnout & Practice Environment & OR 0.71 & $0.68-0.75$ & $<0.05$ \\
\hline & & & & & & Dissatisfaction & Practice Environment & OR 0.60 & $0.57-0.64$ & $<0.05$ \\
\hline & & & & & & Intent to Leave & Practice Environment & OR 0.69 & $0.64-0.75$ & $<0.05$ \\
\hline & & & & & & Poor mgmt. & Practice Environment & OR 0.56 & $0.54-0.59$ & $<0.05$ \\
\hline
\end{tabular}


Table 1. (continued)

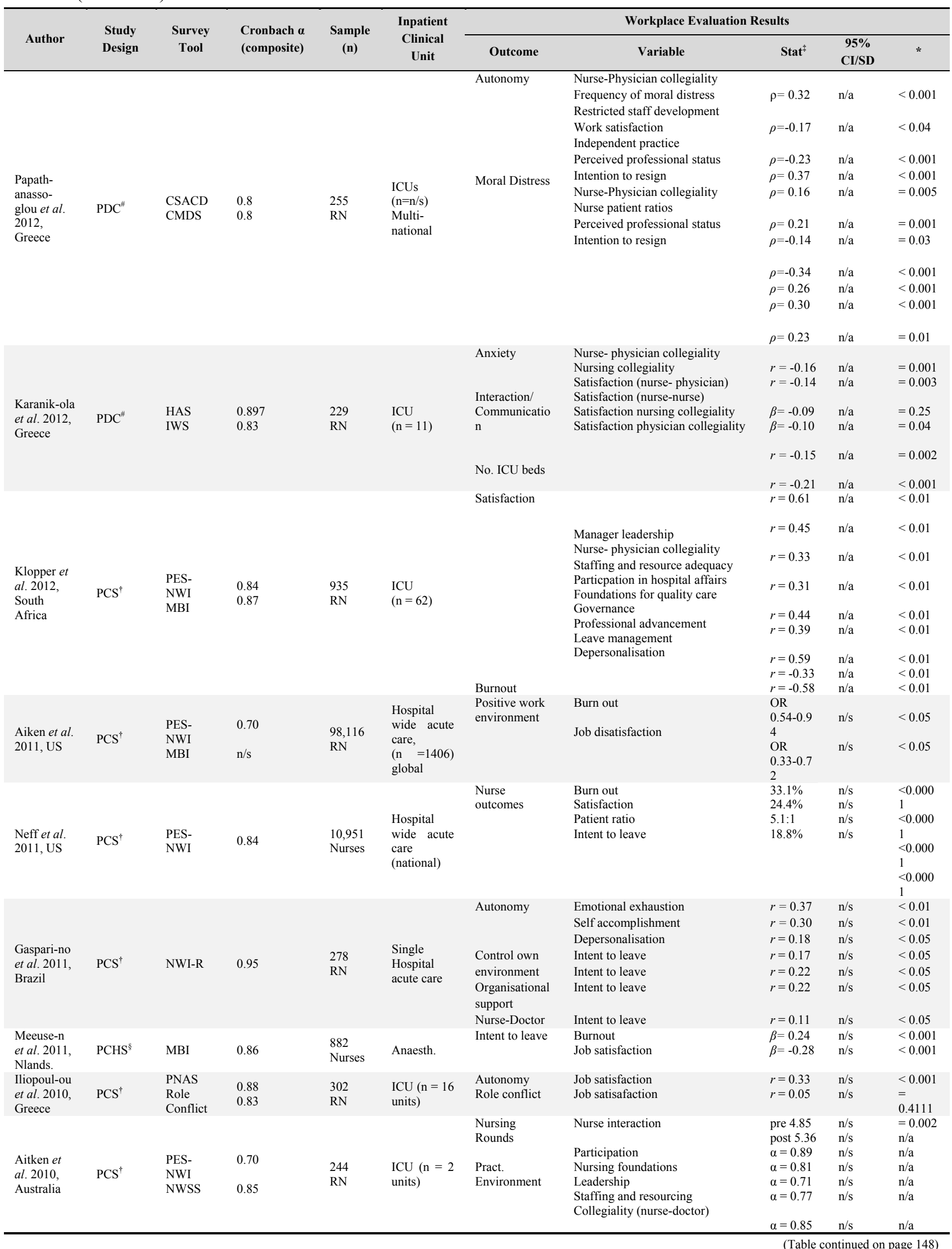


Table 1. (continued)

\begin{tabular}{|c|c|c|c|c|c|c|c|c|c|c|}
\hline \multirow[b]{2}{*}{ Author } & \multirow{2}{*}{$\begin{array}{l}\text { Study } \\
\text { Design }\end{array}$} & \multirow{2}{*}{$\begin{array}{c}\text { Survey } \\
\text { Tool }\end{array}$} & \multirow{2}{*}{$\begin{array}{l}\text { Cronbach } \alpha \\
\text { (composite) }\end{array}$} & \multirow{2}{*}{$\begin{array}{c}\text { Sample } \\
\text { (n) }\end{array}$} & \multirow{2}{*}{$\begin{array}{c}\text { Inpatient } \\
\text { Clinical } \\
\text { Unit }\end{array}$} & \multicolumn{5}{|c|}{ Workplace Evaluation Results } \\
\hline & & & & & & Outcome & Variable & Stat $^{*}$ & $\begin{array}{c}95 \% \\
\text { CI/SD }\end{array}$ & $*$ \\
\hline $\begin{array}{l}\text { Purdy et } \\
\text { al. } 2010, \\
\text { Canada }\end{array}$ & $\mathrm{PCHS}^{\S}$ & $\begin{array}{l}\text { CWEQII } \\
\text { WGCM } \\
\text { NGSQ }\end{array}$ & $\begin{array}{l}0.86 \\
0.78 \\
0.81\end{array}$ & $\begin{array}{l}679 \\
\text { RN }\end{array}$ & $\begin{array}{l}\text { Hospital } \\
\text { wide } \\
\text { acute care } \\
(\mathrm{n}=21 \\
\text { hospitals })\end{array}$ & $\begin{array}{l}\text { Nurse } \\
\text { Empowerment }\end{array}$ & Job satisfaction & $r=0.39$ & $\mathrm{n} / \mathrm{s}$ & $<0.001$ \\
\hline $\begin{array}{l}\text { Roche et } \\
\text { al. } 2010 \text {, } \\
\text { Australia }\end{array}$ & $\begin{array}{l}2^{\text {nd }} \\
\text { analysis } \\
\text { of data }\end{array}$ & $\begin{array}{l}\text { PES- } \\
\text { NWI }\end{array}$ & 0.82 & $\begin{array}{l}2556 \\
\text { Nurses }\end{array}$ & $\begin{array}{c}\text { Acute } \\
\text { care and } \\
\text { mental } \\
\text { health }(\mathrm{n}= \\
26 \\
\text { hospitals) }\end{array}$ & $\begin{array}{l}\text { Practice } \\
\text { environment }\end{array}$ & $\begin{array}{l}\text { Participation } \\
\text { Nursing foundations for } \\
\text { care } \\
\text { Leadership } \\
\text { Staffing and resourcing } \\
\text { Collegiality } \\
\text { (nurse-doctor) }\end{array}$ & $\begin{array}{l}t=4.68 \\
t=-2.81 \\
t=4.06 \\
t=-2.02 \\
t=-6.38\end{array}$ & $\begin{array}{l}\mathrm{n} / \mathrm{s} \\
\mathrm{n} / \mathrm{s} \\
\mathrm{n} / \mathrm{s} \\
\mathrm{n} / \mathrm{s} \\
\mathrm{n} / \mathrm{s}\end{array}$ & $\begin{array}{l}\leq 0.01 \\
\leq 0.01 \\
\leq 0.01 \\
=0.04 \\
\leq 0.01\end{array}$ \\
\hline $\begin{array}{l}\text { Van } \\
\text { Bogaert } \\
\text { et al. } \\
2010 \text {, } \\
\text { Belgium }\end{array}$ & $\mathrm{PCS}^{\dagger}$ & $\begin{array}{l}\text { NWI-R } \\
\text { MBI }\end{array}$ & $\begin{array}{l}0.75 \\
0.83\end{array}$ & $\begin{array}{l}546 \\
\text { RN }\end{array}$ & $\begin{array}{l}\text { Hospital } \\
\text { wide } \\
\text { acute care } \\
(\mathrm{n}=4 \\
\text { hospitals })\end{array}$ & $\begin{array}{l}\text { Job satisfaction } \\
\text { Intention to stay }\end{array}$ & $\begin{array}{l}\text { Collegiality } \\
\text { (nurse-doctor) } \\
\text { Leadership } \\
\text { Organisational support } \\
\text { Collegiality } \\
\text { (nurse-doctor) } \\
\text { Leadership } \\
\text { Organisational support }\end{array}$ & $\begin{array}{l}\text { OR } 3.94 \\
\text { OR } 9.07 \\
\text { OR } 17.2 \\
\text { OR } 2.26 \\
\text { OR } 3.31 \\
\text { OR } 4.65\end{array}$ & $\begin{array}{l}2.90-7.07 \\
3.15-26.2 \\
7.07-72.4 \\
1.23-4.14 \\
0.99-11.2 \\
1.27-17.0\end{array}$ & $\begin{array}{l}<0.0001 \\
<0.0001 \\
<0.0001 \\
<0.05 \\
<0.05 \\
<0.05\end{array}$ \\
\hline $\begin{array}{l}\text { Duffield } \\
\text { et al. } \\
2010 \text {, } \\
\text { Australia }\end{array}$ & $\begin{array}{l}2^{\text {nd }} \\
\text { analysis } \\
\text { of data }\end{array}$ & NWI-R & 0.80 & $\begin{array}{l}2141 \\
\text { Nurses } \\
(1559 \\
\text { RN) }\end{array}$ & $\begin{array}{l}\text { Hospital } \\
\text { wide } \\
\text { acute care } \\
(\mathrm{n}=21 \\
\text { hospitals })\end{array}$ & Intent to leave & $\begin{array}{l}\text { Praise and recognition } \\
\text { Philosophic foundations } \\
\text { Good leadership } \\
\text { Flexible rosters } \\
\text { Participation } \\
\text { Manager visibility } \\
\text { Praise and recognition } \\
\text { Good leadership }\end{array}$ & $\begin{array}{l}\text { OR } 1.47 \\
\text { OR } 1.26 \\
\text { OR } 1.17 \\
\text { OR } 1.16 \\
\text { OR } 1.16 \\
\text { OR } 1.15 \\
\text { OR } 0.83 \\
\text { OR } 0.80\end{array}$ & $\begin{array}{l}1.30-1.67 \\
1.09-1.45 \\
1.03-1.34 \\
1.02-1.30 \\
1.03-1.31 \\
1.03-1.30 \\
0.74-0.94 \\
0.72-0.91\end{array}$ & $\begin{array}{l}<0.01 \\
<0.01 \\
<0.05 \\
<0.05 \\
<0.05 \\
<0.05 \\
<0.01 \\
<0.01\end{array}$ \\
\hline $\begin{array}{l}\text { Cai et al. } \\
2009, \\
\text { China }\end{array}$ & $\mathrm{PCS}^{\dagger}$ & $\begin{array}{l}\text { CWEQII } \\
\text { JAS } \\
\text { ORS }\end{array}$ & $\begin{array}{l}0.82 \\
0.80 \\
0.89\end{array}$ & $\begin{array}{l}189 \\
\text { Staff } \\
\text { Nurses }\end{array}$ & $\begin{array}{l}\text { Hospital } \\
\text { wide } \\
\text { acute care } \\
(\mathrm{n}=2 \\
\text { hospitals })\end{array}$ & $\begin{array}{l}\text { Job satisfaction } \\
\text { Turnover intention }\end{array}$ & $\begin{array}{l}\text { Empowerment } \\
\text { Opportunity } \\
\text { Resources } \\
\text { Organisational support } \\
\text { Empowerment } \\
\text { Formal power (JAS) }\end{array}$ & $\begin{aligned} r & =0.56 \\
r & =-0.22 \\
r & =-0.30 \\
r & =-0.48 \\
r & =-0.31 \\
r & =-0.27\end{aligned}$ & $\begin{array}{l}\mathrm{n} / \mathrm{s} \\
\mathrm{n} / \mathrm{s} \\
\mathrm{n} / \mathrm{s} \\
\mathrm{n} / \mathrm{s} \\
\mathrm{n} / \mathrm{s} \\
\mathrm{n} / \mathrm{s}\end{array}$ & $\begin{array}{l}=0.01 \\
=0.01 \\
=0.01 \\
=0.01 \\
=0.01 \\
=0.05\end{array}$ \\
\hline $\begin{array}{l}\text { Gunnars } \\
\text { dottir } \\
\text { Et al. } \\
2009 \text {, } \\
\text { Iceland }\end{array}$ & $\mathrm{PCS}^{\dagger}$ & $\begin{array}{l}\text { NWI-R } \\
\text { MBI }\end{array}$ & $\begin{array}{l}0.77 \\
0.84\end{array}$ & $\begin{array}{l}695 \\
\text { RN }\end{array}$ & $\begin{array}{l}\text { Hospital } \\
\text { wide } \\
\text { acute care, } \\
(\mathrm{n}=1 \\
\text { hospital })\end{array}$ & $\begin{array}{l}\text { Emotional } \\
\text { exhaustion }\end{array}$ & $\begin{array}{l}\text { Nurse-Physician relations } \\
\text { Unit level support } \\
\text { Staffing } \\
\text { Philosophy of practice } \\
\text { Hospital level support } \\
\text { Nurse-Physician relations } \\
\text { Unit level support } \\
\text { Staffing } \\
\text { Philosophy of practice } \\
\text { Hospital level support }\end{array}$ & $\begin{array}{l}\text { OR } 2.40 \\
\text { OR } 6.70 \\
\text { OR } 2.23 \\
\text { OR } 2.21 \\
\text { OR } 2.95 \\
\beta-2.38 \\
\beta-3.81 \\
\beta-3.95 \\
\beta-2.79 \\
\beta-2.81\end{array}$ & $\begin{array}{l}1.59-3.62 \\
4.10-10.9 \\
1.63-3.05 \\
1.47-3.32 \\
1.93-4.52 \\
\text { SE } 0.63 \\
\text { SE } 0.64 \\
\text { SE } 0.47 \\
\text { SE } 0.65 \\
\text { SE } 0.66\end{array}$ & $\begin{array}{l}<0.001 \\
<0.001 \\
<0.001 \\
<0.001 \\
<0.001 \\
<0.001 \\
<0.001 \\
<0.001 \\
<0.001 \\
<0.001\end{array}$ \\
\hline $\begin{array}{l}\text { Van } \\
\text { Bogaert } \\
\text { et al. } \\
2009 \text {, } \\
\text { Belgium }\end{array}$ & $\mathrm{PCS}^{\dagger}$ & $\begin{array}{l}\text { NWI-R } \\
\text { MBI }\end{array}$ & $\begin{array}{l}0.75 \\
0.83\end{array}$ & $\begin{array}{l}155 \\
\mathrm{RN}\end{array}$ & $\begin{array}{l}\text { Hospital } \\
\text { wide } \\
\text { acute care } \\
(\mathrm{n}=13 \\
\text { hospitals })\end{array}$ & $\begin{array}{l}\text { Organisational } \\
\text { support }\end{array}$ & $\begin{array}{l}\text { Job satisfaction } \\
\text { Intention to leave } \\
\text { Nurse Leadership } \\
\text { Personal accomplishment } \\
\text { Emotional exhaustion } \\
\text { Depersonalisation } \\
\text { Job satisfaction } \\
\text { Intention to leave } \\
\text { Personal accomplishment } \\
\text { Emotional exhaustion } \\
\text { Depersonalisation } \\
\text { Job satisfaction } \\
\text { Intention to leave } \\
\text { Personal accomplishment } \\
\text { Emotional exhaustion } \\
\text { Depersonalisation }\end{array}$ & $\begin{array}{l}\text { OR } 8.80 \\
\text { OR } 5.90 \\
\text { OR } 2.90 \\
\beta 3.20 \\
\beta-3.70 \\
\beta-0.90 \\
\text { OR } 2.90 \\
\text { OR } 1.80 \\
\beta 3.10 \\
\beta-3.30 \\
\beta-1.00 \\
\text { OR } 7.60 \\
\text { OR } 2.90 \\
\beta 2.70 \\
\beta-2.80 \\
\beta-2.40\end{array}$ & $\begin{array}{l}2.60-29.6 \\
1.40-25.0 \\
\mathrm{n} / \mathrm{s} \\
\text { (SE) } 0.8 \\
\text { (SE) } 1.2 \\
\text { (SE) } 0.7 \\
0.90-9.00 \\
1.40-7.60 \\
\text { (SE) } 1.1 \\
\text { (SE) } 1.6 \\
\text { (SE) } 0.9 \\
0.90-65.1 \\
0.30-26.6 \\
\text { (SE } 1.0 \text { ) } \\
\text { (SE } 1.4 \text { ) } \\
\text { (SE } 2.8\end{array}$ & $\begin{array}{l}<0.01 \\
<0.01 \\
<0.01 \\
<0.01 \\
<0.01 \\
\mathrm{n} / \mathrm{s} \\
\mathrm{n} / \mathrm{s} \\
\mathrm{n} / \mathrm{s} \\
<0.01 \\
<0.01 \\
\mathrm{n} / \mathrm{s} \\
\mathrm{n} / \mathrm{s} \\
\mathrm{n} / \mathrm{s} \\
<0.01 \\
<0.01 \\
<0.01\end{array}$ \\
\hline $\begin{array}{l}\text { Aiken et } \\
\text { al. } 2008 \text {, } \\
\text { US }\end{array}$ & $\mathrm{PCS}^{\dagger}$ & $\begin{array}{l}\text { PES- } \\
\text { NWI } \\
\text { MBI }\end{array}$ & $\begin{array}{l}0.79 \\
0.92\end{array}$ & $\begin{array}{l}10,184 \\
\mathrm{RN}\end{array}$ & $\begin{array}{l}\text { Hospital } \\
\text { wide } \\
\text { acute care } \\
(\mathrm{n}=168 \\
\text { hospitals })\end{array}$ & $\begin{array}{l}\text { Care environemnt } \\
\text { Nurse staffing }\end{array}$ & $\begin{array}{l}\text { Burnout } \\
\text { Job satisfaction } \\
\text { Intent to leave }<1 \mathrm{yr} \\
\text { Burnout } \\
\text { Job satisfaction } \\
\text { Intent to leave }<1 \mathrm{yr}\end{array}$ & $\begin{array}{l}\text { OR } .76 \\
\text { OR } 0.75 \\
\text { OR } 0.87 \\
\text { OR } 1.17 \\
\text { OR } 1.11 \\
\text { OR } 1.03\end{array}$ & $\begin{array}{l}0.70-0.82 \\
0.68-0.81 \\
0.79-0.96 \\
1.09-1.25 \\
1.04-1.18 \\
0.95-1.12\end{array}$ & $\begin{array}{l}<0.01 \\
<0.01 \\
<0.01 \\
<0.01 \\
<0.01 \\
<0.10\end{array}$ \\
\hline
\end{tabular}


Table 1. (continued)

\begin{tabular}{|c|c|c|c|c|c|c|c|c|c|c|}
\hline \multirow[b]{2}{*}{ Author } & \multirow{2}{*}{$\begin{array}{l}\text { Study } \\
\text { Design }\end{array}$} & \multirow{2}{*}{$\begin{array}{c}\text { Survey } \\
\text { Tool }\end{array}$} & \multirow{2}{*}{$\begin{array}{c}\text { Cronbach } \\
\alpha \\
\text { (composite) }\end{array}$} & \multirow{2}{*}{$\begin{array}{c}\text { Sample } \\
\text { (n) }\end{array}$} & \multirow{2}{*}{$\begin{array}{c}\text { Inpatient } \\
\text { Clinical } \\
\text { Unit }\end{array}$} & \multicolumn{5}{|c|}{ Workplace Evaluation Results } \\
\hline & & & & & & Outcome & Variable & Stat ${ }^{\ddagger}$ & $\begin{array}{c}95 \% \\
\text { CI/SD }\end{array}$ & * \\
\hline $\begin{array}{l}\text { Faulkner } \\
\text { et al. } \\
2008, \\
\text { Canada }\end{array}$ & $\mathrm{PCS}^{\dagger}$ & $\begin{array}{l}\text { CWEQ-II } \\
\text { PEQ } \\
\text { ERIQ }\end{array}$ & $\begin{array}{l}0.80 \\
0.89 \\
0.77\end{array}$ & $\begin{array}{l}282 \\
\mathrm{RN}\end{array}$ & $\begin{array}{l}\text { Hospital } \\
\text { wide } \\
\text { acute care } \\
\text { (n=168 } \\
\text { hospitals) }\end{array}$ & $\begin{array}{l}\text { Prof. } \\
\text { Respect }\end{array}$ & $\begin{array}{l}\text { Structural empowerment } \\
\text { Informal power } \\
\text { Support } \\
\text { Formal power } \\
\text { Resources } \\
\text { Information } \\
\text { Opportunity } \\
\text { Psychological empowerment } \\
\text { Autonomy } \\
\text { Impact } \\
\text { Meaning }\end{array}$ & $\begin{aligned} r & =0.47 \\
r & =0.44 \\
r & =0.38 \\
r & =0.34 \\
r & =0.32 \\
r & =0.30 \\
r & =0.24 \\
r & =0.32 \\
r & =0.31 \\
r & =0.25 \\
r & =0.22\end{aligned}$ & $\begin{array}{l}\mathrm{n} / \mathrm{a} \\
\mathrm{n} / \mathrm{a} \\
\mathrm{n} / \mathrm{a} \\
\mathrm{n} / \mathrm{a} \\
\mathrm{n} / \mathrm{a} \\
\mathrm{n} / \mathrm{a} \\
\mathrm{n} / \mathrm{a} \\
\mathrm{n} / \mathrm{a} \\
\mathrm{n} / \mathrm{a} \\
\mathrm{n} / \mathrm{a} \\
\mathrm{n} / \mathrm{a}\end{array}$ & $\begin{array}{l}<0.05 \\
<0.05 \\
<0.05 \\
<0.05 \\
<0.05 \\
<0.05 \\
<0.05 \\
<0.05 \\
<0.05 \\
<0.05 \\
<0.05\end{array}$ \\
\hline $\begin{array}{l}\text { Manojlo- } \\
\text { vich et } \\
\text { al. } 2008 \text {, } \\
\text { US }\end{array}$ & $\mathrm{PCS}^{\dagger}$ & ICU-NPQ & 0.85 & $\begin{array}{l}462 \\
\mathrm{RN}\end{array}$ & $\begin{array}{l}\text { ICU }(n= \\
25 \text { units })\end{array}$ & $\begin{array}{l}\text { Job } \\
\text { satisfaction }\end{array}$ & Nurse-Doctor communication & $r=0.34$ & $\mathrm{n} / \mathrm{a}$ & $<0.001$ \\
\hline $\begin{array}{l}\text { Lai et al. } \\
2008, \\
\text { Taiwan }\end{array}$ & $\mathrm{PCS}^{\dagger}$ & $\begin{array}{l}\text { Locally } \\
\text { Developed } \\
\text { (Coopers) }\end{array}$ & 0.84 & $\begin{array}{l}130 \\
\mathrm{RN}\end{array}$ & $\begin{array}{l}\text { ICU } \\
(\mathrm{n}=2 \\
\text { units })\end{array}$ & $\begin{array}{l}\text { Intention to } \\
\text { leave }\end{array}$ & $\begin{array}{l}\text { Level of happiness } \\
\text { Depression } \\
\text { Job satisfaction }\end{array}$ & $\begin{array}{ll}\bar{x} & 2.27 \\
\bar{x} & 4.25 \\
\bar{x} & 6.75\end{array}$ & $\begin{array}{l}\text { SD } 0.85 \\
\text { SD } 1.85 \\
\text { SD } 1.61\end{array}$ & $\begin{array}{l}<0.01 \\
<0.01 \\
<0.01\end{array}$ \\
\hline $\begin{array}{l}\text { Stordeur } \\
\text { et al. } \\
2007, \\
\text { Belgium }\end{array}$ & $\mathrm{PCS}^{\dagger}$ & $\begin{array}{l}\text { NEXT } \\
\text { NSS } \\
\text { COPSOQ } \\
\text { ERIQ }\end{array}$ & $\begin{array}{l}0.86 \\
0.74 \\
0.84 \\
0.72\end{array}$ & $\begin{array}{l}1175 \\
\text { RN }\end{array}$ & $\begin{array}{l}\text { Hospital } \\
\text { wide } \\
\text { acute care } \\
(\mathrm{n}=12 \\
\text { hospitals) }\end{array}$ & $\begin{array}{l}\text { Organisation } \\
\text { climate }\end{array}$ & $\begin{array}{l}\text { Schedule/roster flexibility } \\
\text { Workload } \\
\text { Emotional exhaustion } \\
\text { Role ambiguiy } \\
\text { Nursing management } \\
\text { Nursing team communication } \\
\text { Job satisfaction } \\
\text { Burnout } \\
\text { Intention to leave }\end{array}$ & $\begin{array}{l}\bar{x} 4.2 \\
\bar{x} 3.0 \\
\bar{x} 3.3 \\
\bar{x} 2.2 \\
\bar{x} 3.1 \\
\bar{x} 3.8 \\
\bar{x} 3.5 \\
\bar{x} 2.2\end{array}$ & $\begin{array}{l}\mathrm{n} / \mathrm{s} \\
\mathrm{n} / \mathrm{s} \\
\mathrm{n} / \mathrm{s} \\
\mathrm{n} / \mathrm{s} \\
\mathrm{n} / \mathrm{s} \\
\mathrm{n} / \mathrm{s} \\
\mathrm{n} / \mathrm{s} \\
\mathrm{n} / \mathrm{s} \\
\mathrm{n} / \mathrm{s}\end{array}$ & $\begin{array}{l}<0.001 \\
<0.001 \\
<0.001 \\
<0.001 \\
<0.001 \\
<0.001 \\
<0.001 \\
<0.001 \\
<0.001\end{array}$ \\
\hline $\begin{array}{l}\text { Stone et } \\
\text { al. } 2006 \text {, } \\
\text { US }\end{array}$ & $\mathrm{PCS}^{\dagger}$ & $\begin{array}{l}\text { PNWE } \\
\text { (NWI-R) }\end{array}$ & 0.78 & $\begin{array}{l}2323 \\
\mathrm{RN}\end{array}$ & $\begin{array}{l}\text { ICU } \\
(\mathrm{n}=110 \\
\text { units/66 } \\
\text { hospitals) }\end{array}$ & $\begin{array}{l}\text { Intention to } \\
\text { leave }\end{array}$ & $\begin{array}{l}\text { Professional practice } \\
\text { Nursing management } \\
\text { Staffing and resource } \\
\text { adequacy } \\
\text { Nursing process } \\
\text { Nurse-Doctor collegiality } \\
\text { Nursing competence } \\
\text { Positive scheduling climate } \\
\text { Professional practice } \\
\text { Nursing management } \\
\text { Staffing and resource adequac } \\
\text { Nursing process } \\
\text { Nurse-Doctor collegiality } \\
\text { Nursing competence } \\
\text { Positive scheduling climate } \\
\text { Bed size (small) } \\
\text { Bed size (medium }\end{array}$ & $\begin{array}{l}\bar{x} 2.20 \\
\bar{x} 2.24 \\
\bar{x} 2.77 \\
\bar{x} 2.34 \\
\bar{x} 2.51 \\
\bar{x} 2.92 \\
\bar{x} 2.48 \\
\text { OR } 0.52 \\
\text { OR } 0.74 \\
\text { OR } 1.23 \\
\text { OR } 0.81 \\
\text { OR } 1.31 \\
\text { OR } 0.61 \\
\text { OR } 0.81 \\
\text { OR } 1.00\end{array}$ & $\begin{array}{l}\text { SE } 0.08 \\
\text { SE } 0.08 \\
\text { SE } 0.06 \\
\text { SE } 0.06 \\
\text { SE } 0.06 \\
\text { SE } 0.07 \\
\text { SE } 0.09 \\
0.42-0.64 \\
0.51-1.08 \\
0.88-1.72 \\
0.54-1.20 \\
0.85-2.03 \\
0.44-0.83 \\
0.60-1.11 \\
0.61-1.63 \\
0.78-1.88\end{array}$ & $\begin{array}{l}<0.001 \\
<0.001 \\
<0.001 \\
<0.001 \\
<0.001 \\
<0.001 \\
<0.001 \\
<0.01 \\
\mathrm{n} / \mathrm{s} \\
\mathrm{n} / \mathrm{s} \\
\mathrm{n} / \mathrm{s} \\
\mathrm{n} / \mathrm{s} \\
<0.01 \\
\mathrm{n} / \mathrm{s} \\
\mathrm{n} / \mathrm{s} \\
\mathrm{n} / \mathrm{s}\end{array}$ \\
\hline $\begin{array}{l}\text { Manojlo- } \\
\text { vich et } \\
\text { al. } 2005 \text {, } \\
\text { US }\end{array}$ & $\mathrm{PCS}^{\dagger}$ & $\begin{array}{l}\text { CWEQ-II } \\
\text { PES-NWI } \\
\text { IWS }\end{array}$ & $\begin{array}{l}0.90 \\
0.93 \\
0.92\end{array}$ & $\begin{array}{l}284 \\
\mathrm{RN}\end{array}$ & $\begin{array}{l}\text { Hospital } \\
\text { wide } \\
\text { acute care } \\
(\mathrm{n}=\mathrm{n} / \mathrm{s}) \\
\text { Multi. }\end{array}$ & $\begin{array}{l}\text { Structural } \\
\text { empowerment } \\
\text { Practice } \\
\text { environment } \\
\text { Job } \\
\text { satisfaction }\end{array}$ & $\begin{array}{l}\text { Nurse-Doctor communcation } \\
\text { Job satisfaction } \\
\text { Nurse-Doctor communication } \\
\text { Job satisfaction } \\
\text { Nurse-Doctor communication } \\
\text { Structural empowerment }\end{array}$ & $\begin{array}{l}\beta 0.27 \\
\beta 0.22 \\
\beta 0.22 \\
\beta 0.39 \\
\beta 0.37 \\
\beta 0.22\end{array}$ & $\begin{array}{l}\mathrm{n} / \mathrm{s} \\
\mathrm{n} / \mathrm{s} \\
\mathrm{n} / \mathrm{s} \\
\mathrm{n} / \mathrm{s} \\
\mathrm{n} / \mathrm{s} \\
\mathrm{n} / \mathrm{s}\end{array}$ & $\begin{array}{l}\leq 0.05 \\
\leq 0.05 \\
\leq 0.05 \\
\leq 0.05 \\
\leq 0.05 \\
\leq 0.05\end{array}$ \\
\hline $\begin{array}{l}\text { Minviell } \\
\text {-e et al. } \\
2005 \\
\text { France }\end{array}$ & $\mathrm{PCS}^{\dagger}$ & $\begin{array}{l}\mathrm{OCI} \\
\mathrm{MBI}\end{array}$ & $\begin{array}{l}>0.70 \\
>0.70\end{array}$ & $\begin{array}{l}1000 \\
(\mathrm{RN} \\
750)\end{array}=$ & $\begin{array}{l}\text { Hospital } \\
\text { wide } \\
\text { acute care } \\
(\mathrm{n}=\mathrm{n} / \mathrm{s}) \\
\text { Mutli. }\end{array}$ & $\begin{array}{l}\text { Job } \\
\text { satisfaction }\end{array}$ & $\begin{array}{l}\text { Participation (affiliation) } \\
\text { Empowerment } \\
\text { Competence } \\
\text { Achievement } \\
\text { Self actualising }\end{array}$ & $\begin{aligned} r & =0.36 \\
r & =-0.11 \\
r & =0.02 \\
r & =0.25 \\
r & =0.36\end{aligned}$ & $\begin{array}{l}\mathrm{n} / \mathrm{s} \\
\mathrm{n} / \mathrm{s} \\
\mathrm{n} / \mathrm{s} \\
\mathrm{n} / \mathrm{s} \\
\mathrm{n} / \mathrm{s}\end{array}$ & $\begin{array}{l}<0.001 \\
<0.01 \\
<0.001 \\
<0.001 \\
<0.001\end{array}$ \\
\hline
\end{tabular}

Note. $\ddagger$ Statistic legend: * significance; $\rho=$ Pearsons Correlation coefficient; $r=$ sample correlation coefficient; $\beta=$ regression coefficient; OR $=$ odds ratio; $\mathrm{t}=\mathrm{t}$-test; $\bar{x}=$ mean; ${ }^{\dagger}$ PCS $=$ Prospective Cross Sectional Survey; ${ }^{\#}$ PDC $=$ Prospective Descriptive Correlation; ${ }^{8}$ PCHS $=$ Prospective Cohort Study; $\mathrm{n} / \mathrm{a}=$ not applicable; $\mathrm{n} / \mathrm{s}=$ not specified.

Thirty one criteria were used to derive a quality index score for each study. Potential study bias was assessed using the risk assessment process adapted from a Cochrane Systematic Review undertaken by Inglis et al. (2010) ${ }^{[40]}$. The highest composite score attainable was seventy seven. Each score was then converted to percentages to assess the relative quality for each study (see Figure 2).

The mean quality index score was $85 \%$ with minimal variability in the range (75\%-91\%). Highest scores reflected multicenter studies with a large sample size, clearly defined outcome measures, demonstrated survey instrument validation, high survey response rate, identified complex associations within the results and demonstrated relevance to health services management ${ }^{[11,42]}$. Conversely, the lowest scoring study was conducted in a single site with a small 
convenience sample, and implications for practice were not clearly articulated limiting broader generalization of results ${ }^{[43]}$.

All studies, except one ${ }^{[42]}$, failed to explicitly define the study population exclusion criteria potentially affecting sample selection, with the majority using a convenience sample. While this may limit generalization of results, sample sizes were considered to be moderate to large (range $\mathrm{n}=67$ to 98,116 ), mitigating this risk.

Figure 2. Relative derived quality index scores

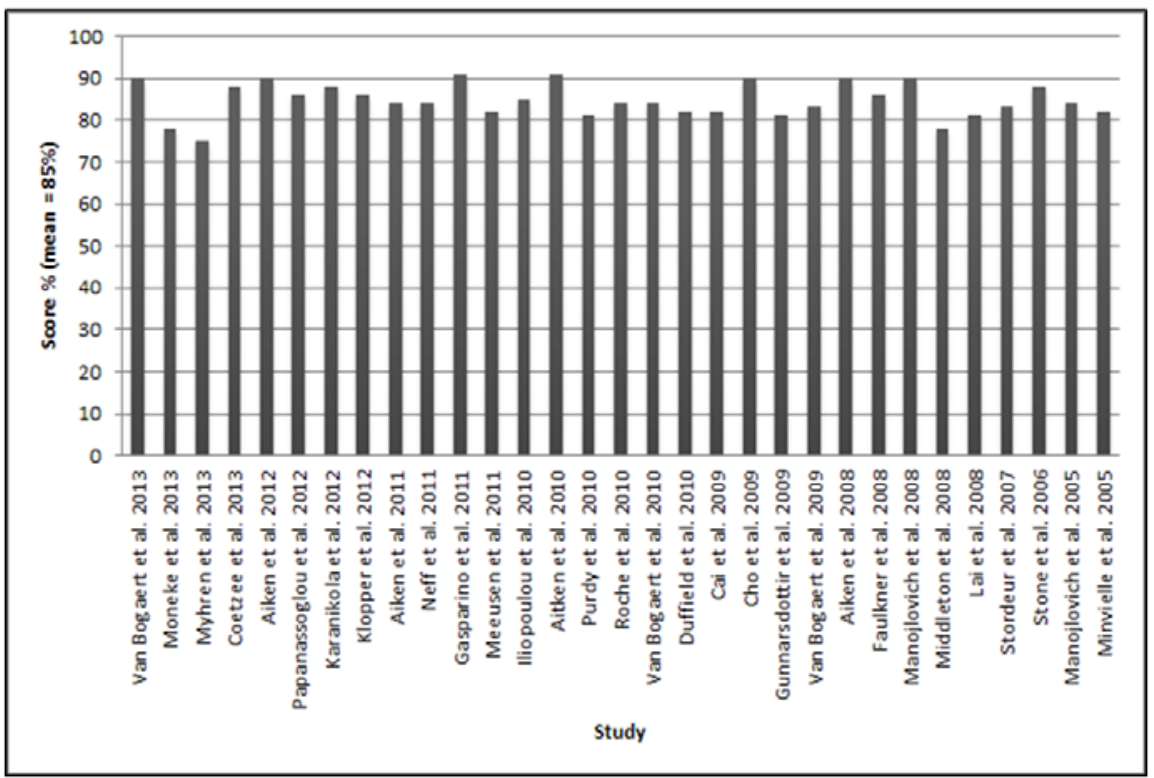

Seven studies also employed randomization to control for confounding ${ }^{[3,26,44-47]}$. Overall the studies were primarily multi-site from a broad range of countries with two being multi-national ${ }^{[45,48]}$. All studies were undertaken in an acute care environment with nine studies specific to adult ICU. A majority of studies were strong in terms of author expertise, clear study purpose, prospective study design and using psychometrically validated survey instruments. Results were comprehensively reported using clear descriptive summaries, empirical statistical analysis and identification of significant associations between structural characteristics of the workplace environment and nurse outcomes. These results were then further qualified through reporting of small standard errors, standard deviations and/or narrow confidence intervals. Overall the quality of the studies was high (see Figure 2) further supporting the inclusion of the twenty one identified nurse outcomes in the minimum dataset.

\subsection{Data abstraction and synthesis}

At the data analysis stage the authors followed the sequence proposed by Whittemore and Knafl (2005) including data reduction, data display, data comparison and verification of conclusions ${ }^{[25]}$. The data were reduced by extraction of nurse outcome measures as summarized in Table 1. This enabled a systematic identification of nurse outcomes associated with organizational factors from the described statistical testing, associations and conclusions. Nurse outcomes were reduced to a minimum dataset against which the survey instruments were aligned to assess the degree of congruence with the outcomes collected by each instrument.

\section{Conclusion}

Systematic appraisal found overall a high level of study quality in terms of research methodology and reporting. This provided the reviewers with confidence regarding the validity of nurse outcome measures identified. Further analysis of individual outcome measures was undertaken to statistically validate the final dataset of nurse outcome measures used to select an appropriate survey instrument. 


\section{Results}

\subsection{Nurse outcomes associated with organizational structures in the work environment}

Repeated testing across multiple studies supports the reliability of nurse outcome measures. Figure 3 illustrates the frequency each nurse outcome was measured. Job satisfaction, intention to leave, leadership, emotional exhaustion (burnout), resourcing and staffing, and Nurse-Doctor collegiality were frequently used to study nurse work environments. In contrast, professional advancement and satisfaction with nursing in general were measured only once in separate large multicenter studies with high relative quality index scores ${ }^{[48,49]}$. All twenty one nurse outcome measures were therefore retained for further evaluation in order of highest to lowest frequency.

Figure 3. Repeatability of nurse outcome measures

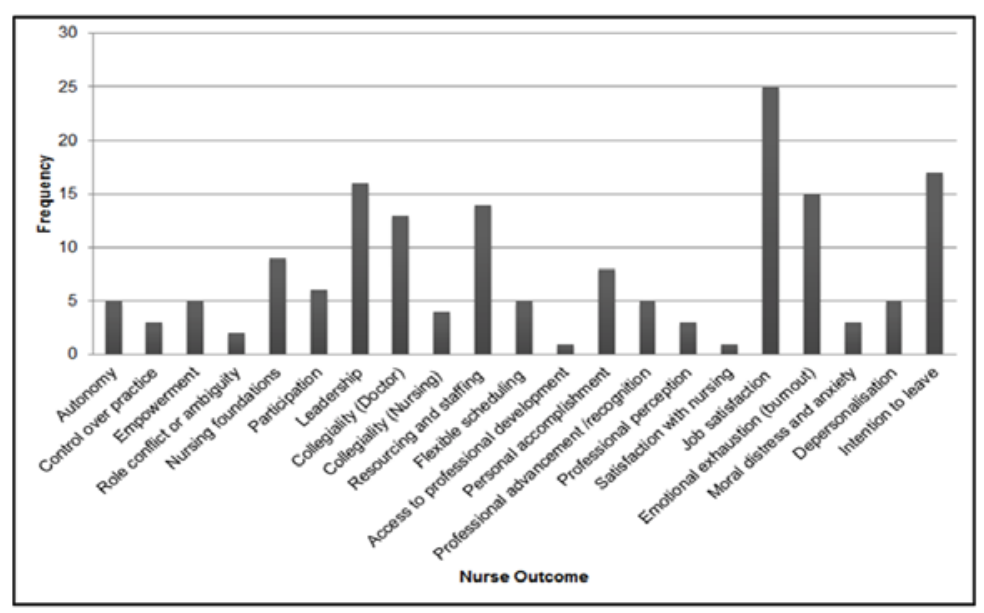

\subsubsection{J ob satisfaction}

Job satisfaction was strongly associated with the work environment in twenty five studies, including seven in ICU, with particular influence on intention to leave $(\bar{x}=-4.25 ; S D 1.61 ; p<.01)^{[50]}$ and $(\beta=-0.28 ; p<.001)^{[51]}$. A study of 935 ICU nurses identified a positive association between job satisfaction and nurse leadership $(r=0.612 ; p<.001)$, nurse-physician collegiality $(r=0.454 ; p<.001)$, staffing and resource adequacy $(r=0.328 ; p<.001)$, participation $(r=0.307 ; p<.001)$, foundations for quality care $(r=0.437 ; p<.001)$ and professional advancement $(r=0.595 ; p<.001)^{[52]}$. Job satisfaction was also found to have a significant correlation with increased autonomy $(r=0.331 ; p<.001)$ in a study of 431 ICU nurses ${ }^{[53]}$.

\subsubsection{Intention to leave}

Seventeen studies, four in ICU, measured intention to leave. One large prospective study of 2323 ICU nurses found associations between intention to leave and professional status $(\bar{x}=2.20$, SE $0.08 ; p<.001)$, nursing leadership $(\bar{x}=$ 2.24, SE 0.08; $p<.001)$, staffing and resource adequacy $(\bar{x}=2.27$, SE $0.06 ; p<.001)$, nursing foundations $(\bar{x}=2.34$, SE $0.06 ; p<.001)$, nurse-physician collegiality $(\bar{x}=2.51$, SE $0.06 ; p<.001)$ and rostering flexibility $(\bar{x}=2.48$, SE 0.09 ; $p<.001)^{[3]}$. These associations were also found two ICU studies ${ }^{[48,54]}$ and five studies in acute care settings ${ }^{[42,46,51,55,56]}$.

\subsubsection{Leadership}

Nursing leadership repeatedly demonstrated significant impact on job satisfaction, participation, retention and perceived professional status. Sixteen studies underscored the importance good nurse leadership with four studies conducted in ICU ${ }^{[3,41,57]}$. Stone et al. (2006) ${ }^{[3]}$ identified that leadership in ICU was significantly associated with intention to leave $(\bar{x}=2.28$, SE $0.08 ; p<.001)$ while Klopper et al. $(2012)^{[57]}$ found a moderately strong correlation between leadership and a positively perceived ICU workplace $(r=0.612 ; p<.01)$. The bulk of the studies were conducted in non-ICU acute care environments. A large Australian multicenter study of 1,559 nurses identified a significant association between good 
clinical leadership and improved job satisfaction (OR 1.17; 95\% CI 1.03-1.34; $p<.05$ ), and reduced intention to leave (OR $0.80 ; 95 \%$ CI $0.72-0.91 ; p<.01)^{[49]}$.

\subsubsection{Emotional exhaustion}

Emotional exhaustion was explored in fifteen studies, three in ICU ${ }^{[50,52,54]}$. A significant association was consistently reported between the level of emotional exhaustion, or burn out, by nursing staff. The most frequently reported significant contributing factors to emotional exhaustion were staffing (OR 1.17, 95\% CI $1.09-1.25 ; p<.01)^{[44]}$ and (OR 050, 95\% CI $0.34-0.73 ; p<.005)^{[54]}$, sense of depersonalization $(r=-0.576 ; p<.01)^{[52]}$ and professional perception of nurses (OR 0.76 , $95 \%$ CI $0.70-0.82 ; p<.001)^{[44]}$. A recent study concluded that emotional exhaustion is an important predictor of a broad range of nurse outcomes ${ }^{[56]}$.

\subsubsection{Resourcing and staffing}

Fourteen studies found a significant association between perception of adequate resourcing and the work environment, with four studies conducted in ICU ${ }^{[3,48,52,54]}$. A moderately strong correlation was also found with job satisfaction $(r=$ $0.328 ; p<.01$ ), while intention to leave (OR $1.23 ; 95 \% 0.88-1.72$ ) was not statistically significant ${ }^{[57]}$. More broadly, in nine non-ICU studies, inadequate staffing and resourcing was associated with nurses having a negative perception of the work environment, including a large Australian study of 2,556 nurses $(t=-2.02 ; p=.04)^{[60]}$.

\subsubsection{Nurse-doctor collegiality}

Effective Nurse-Doctor collegiality repeatedly influenced perception of the workplace environment. Thirteen studies found a significant association between Nurse-Doctor collaboration and nurse autonomy, emotional exhaustion and anxiety, job satisfaction and satisfaction with nursing generally, with five of these studies conducted in ICU ${ }^{[3,41,46,48,52]}$. Of note is a study of 935 ICU nurses finding that Nurse-Doctor collegiality had a moderately strong correlation with job satisfaction $(r=0.454 ; p<.01)^{[52]}$. Staff also expressed a higher sense autonomy $(r=0.319 ; p<.001)^{[48]}$; job satisfaction (OR 3.94; 95\% CI 2.90-7.07; $p<.0001)^{[61]}$ and $(r=0.34 ; p<.001)^{[62]}$; and nurse empowerment $(\beta=0.27 ; p<.05)^{[26]}$ when Nurse-Doctor collegiality was high. Conversely, a number of studies found increased intention to leave associated with low collegiality $(r=0.11 ; p<.05)^{[42]}$, (OR 2.26, 95\% CI 1.23-4.14; $\left.p<.05\right)^{[61]}$ and $(\bar{x}=2.51 \text {, SE } 0.06, p<.001)^{[3]}$.

\subsubsection{Nursing foundations for quality care}

High quality care, underpinned by a nursing foundation based on a defined nursing philosophy and nursing model of care, was found to be associated with a positive working environment in nine studies, three of which were conducted in $\mathrm{ICU}^{[3,41,52]}$. Typically this was manifested by increased job satisfaction both in $\operatorname{ICU}(r=0.437 ; p<.01){ }^{[52]}$ and in acute care areas (OR 1.26, 95\% CI 1.09-1.45; $p<.01)^{[63]}$.

\subsubsection{Personal accomplishment}

A perception of higher personal accomplishment was associated with a positive work environment in eight studies, one of which undertaken in ICU ${ }^{[3]}$. Perceptions of high nurse autonomy $(r=0.30 ; p<.01)^{[42]}$ and professional respect $(r=0.32$; $p<.05)^{[64]}$, and increased job satisfaction $(r=0.36 ; p<.001)^{[65]}$ were evident when the sense of personal accomplishment was high. This positive association was also found where there was effective Nurse-Doctor collegiality $(\beta=3.20$, SE 0.8; $p<.01)$, strong leadership $(\beta=3.10$, SE $1.1 ; p<.01)$ and organizational support $(\beta=2.70$, SE $1.0 ; p<.01){ }^{[61]}$. ICU nurses reported a higher intention to leave where they perceived a lack of personal accomplishment $(\bar{x}=2.92$, SE 0.07; $p<.001)^{[3]}$.

\subsubsection{Nurse participation}

Increased participation in hospital affairs was associated with a positive work environment in six studies, with two conducted in ICU ${ }^{[41,52]}$. Job satisfaction increased with higher participation $(r=0.307 ; p<.01){ }^{[52]}$, (OR 1.16; 95\% CI $1.03-1.31 ; p<.05){ }^{[63]}$ and $(r=0.36 ; p<.001)^{[65]}$. Hospitals achieving magnet status typically have higher rates of participation $(t=4.68 ; p<.01)^{[60]}$ and $(\bar{x}=2.76, S D 0.44 ; p<.001)^{[66]}$. 


\subsubsection{Depersonalization}

The perception of being depersonalized from the work environment was identified as a strong predictor of emotional exhaustion and job satisfaction in three acute care studies ${ }^{[2,47,56]}$ and two in ICU ${ }^{[43,52]}$. Perceived depersonalization had a moderate inverse association with reduced job satisfaction in a study of 129 ICU nurses $(r=-0.313 ; p<.001){ }^{[43]}$.

\subsubsection{Professional recognition}

Five studies consistently identified perceived professional recognition as a key nurse outcome, with one study conducted in ICU ${ }^{[52]}$. Professional recognition was found to increase nurses' job satisfaction in ICU $(r=0.595 ; p<.01)^{[52]}$ and in acute care areas (OR 1.47; 95\%CI 2.90-7.07; $p<.01)^{[63]}$ and $(r=0.25 ; p<.001)^{[65]}$. Professional recognition positively influences the perception of professional respect $(r=0.24 ; p<.05)^{[64]}$.

\subsubsection{Nurse autonomy}

Perceived autonomy was found to be an important a nurse outcome measure in five studies, with two specific to ICU ${ }^{[48,53]}$. In the largest prospective study of $431 \mathrm{ICU}$ nurses increased job satisfaction had a moderate correlation with increased autonomy $(r=0.331 ; p<.001){ }^{[53]}$ which was supported in a later study $(r=0.369 ; p<.001){ }^{[48]}$. ICU nurses also perceived higher autonomy when there was effective Nurse-Doctor collegiality $(r=0.319 ; p<.001)$, access to staff development $(r=0.369 ; p<.001)$ and perceived professional recognition $(r=0.211 ; p=.001)^{[48]}$ and $(r=0.31 ; p<.05)^{[64]}$. Higher levels of emotional exhaustion $(r=0.37 ; p<0.01)$ and perceived depersonalization $(r=0.18 ; p<.05)$ were associated with reduced autonomy as was low self-accomplishment $(r=0.30 ; p<.01)^{[42]}$. Intention to leave was also influenced by lower perceived autonomy $(r=-0.142 ; p=.03)^{[48]}$.

\subsubsection{Nurse empowerment}

A perception of increased empowerment was associated with a positive work environment in five studies conducted in acute care areas. Where nurses perceived increased empowerment job satisfaction was increased $(r=0.39 ; p<.001)^{[67]}$, $(r=0.56 ; p=.01)^{[68]},(\beta=0.22 ; p<.05)^{[26]}$ and $(r=-0.11 ; p<.01)^{[65]}$. Empowerment increased with professional respect $(r=0.39 ; p<.001)^{[64]}$ and effective Nurse-Doctor collegiality $(\beta=0.27 ; p<.05)^{[26]}$, and was low when intention to leave was expressed $(r=-0.31 ; p=.01)^{[68]}$.

\subsubsection{Flexible rostering}

Five studies identified flexible rostering as a determinant of a positive work environment, two of which were conducted in $\mathrm{ICU}^{[3,52]}$. Rostering inflexibility increases emotional exhaustion $(r=-0.325 ; p<.01)^{[52]}$ and intention to leave $(\bar{x}=2.48$, SE $0.09, p<.001)^{[3]}$. Organizational climate is rated higher $(\bar{x}=4.2 v s .3 .8 ; p<.001)^{[69]}$ and job satisfaction increases with flexible rostering (OR 1.16; 95\%CI 1.02-1.30; $p<.05)^{[63]}$.

\subsubsection{Nurse-nurse communication}

Four studies investigated nurse-nurse communication in the workplace, with two conducted in ICU ${ }^{[41,46]}$. Improved communication attributed to introducing formalized ICU nursing rounds improved perceptions of the workplace $(\bar{x}=$ 4.85 vs. post $\bar{x}=5.36 ; p=.002)^{[41]}$, while poor communication decreased job satisfaction $(\beta=-0.097 ; p=.04)$ and compounded self-rated anxiety $(\mathrm{r}=-0.160 ; p=.001)^{[46]}$. The organizational climate benefited from improved nursing communication $(\bar{x}=3.8 v s . \bar{x}=3.3 ; p<.001)^{[57]}$ and interestingly the higher the number of ICU beds the lower the rating of effective nurse communication $(r=-0.152 ; p=.002)^{[46]}$. This might be postulated to be associated with a large nursing workforce and depersonalization in larger ICUs. Further to this observation, though not statistically significant, was an increased intention to leave in larger capacity ICUs (OR 1.21; 95\% CI $0.78-1.88 ; p<.05)^{[3]}$.

\subsubsection{Nurse outcome measures with limited supporting evidence}

Three nurse outcome measures were identified that were supported by three studies or less. These outcomes, however, are consistent with recommended professional standards for healthy work environments and merit consideration ${ }^{[70]}$. Increased control over practice is associated with greater autonomy $(r=0.159 ; p=.005)^{[48]}$ and where an inability to control practice 
exists this is associated with increased intention to leave $(r=-0.22 ; p<.05)^{[42]}$. Moral distress was also found to increase where poor Nurse-Doctor collegiality existed $(r=-0.337 ; p<.001)$ and with increased intention to leave $(r=0.229$; $p=.01)^{[48]}$.

\subsubsection{Conclusion}

Twenty one nurse outcomes in acute and intensive care work environment were identified and evaluated using the following steps: (1) assessing the quality of the relevant primary study and generating a quality index score; (2) assessing the risk of bias for each primary study; (3) examining the significance of the association between nurse outcome measures and structural features of the workplace environment; and (4) evaluating the repeatability and consistency of nurse outcome measures. Following this process all twenty one nurse outcome measures were retained to inform selection of a nurse survey instrument for ICU.

\section{Discussion and instrument selection}

Internationally, professional nursing associations recommend standards for healthy work environments that promote the balance of an organization's objectives with favorable nurse outcomes ${ }^{[71,72]}$. Where this balance is achieved magnet health care organizations evolve characterized by high quality nursing care, increased job satisfaction and improved nurse outcomes ${ }^{[66,73,74]}$.

Magnet organizations value nursing practice, workplace culture and climate, as well as material factors such as rates of pay ${ }^{[74-76]}$. Strong leadership is a key factor and is considered to influence job satisfaction, participation levels, staff retention and perceived professional status ${ }^{[77-79]}$.

Healthy work environments recognize strong nursing foundations, active staff participation, empowerment and team building as a basis for high quality care ${ }^{[80,81]}$. An effective ICU clinical team is further underpinned by a high level of Nurse-Doctor collegiality to sustain a positive organizational culture and climate ${ }^{[82-84]}$.

Dissatisfaction and worsening staff outcomes are associated with health service restructuring aimed at improving productivity through work intensification ${ }^{[85-87]}$. Staff outcomes are also influenced by rostering, poor physician-nurse interactions, new technology, staff shortages, unpredictable work flow, lack of control over practice and a perception that patient care is not coordinated, evidence-based or unsafe ${ }^{[39,48,49]}$.

Job satisfaction is associated consistently with positive work environment characteristics including nurse autonomy, staffing and resourcing, opportunities for professional advancement and positive acknowledgement ${ }^{[32]}$. Intention to leave is reduced and job satisfaction is high where staff perceive they have equitable rosters, flexibility and control over personal time ${ }^{[16,88]}$.

Structural and psycho-sociological factors determine nurse outcomes making it essential that both are appropriately captured in organizational survey instruments. High interdependence exists between organizational, interpersonal and individual behavior determinants of a health work environment ${ }^{[86]}$.

Perceptions held by nurses on how structural factors impact on them personally and may be manifested as emotional exhaustion ${ }^{[16]}$. Emotional exhaustion refers to the depletion of aroused emotional states, such as a nurse feeling too emotionally drained to adequately care for patients. Combined with a sense of low personal accomplishment and depersonalization then these perceptions are manifested as 'burn-out' and increased intention to leave ${ }^{[89]}$.

Lack of personal accomplishment is linked to an individual's lower perception of self-competence and empowerment ${ }^{[90]}$. Empowerment is an important component of transformational leadership and the trust underpinning staff autonomy and 
job satisfaction ${ }^{[91]}$. Effective communication supports control over practice, decision-making at the bedside and teamwork, all determinants of a positive workplace and ultimately a positive work environment ${ }^{[48,92]}$.

Nurse outcomes reflect external structural factors and individual perceptions both of which are influenced by the work environment as recognized in professional standards and magnet hospitals ${ }^{[70,74,93]}$. The most appropriate survey instrument should capture the impact of structural factors and individual perceptions and thereby align closely with the nurse outcome dataset identified.

Repeated testing of instruments over time in similar nurse populations provides an indication of their reproducibility and reliability. Taking into account the level of instrument congruence with the nurse outcome dataset, evidence of content and contextual validity and the frequency of testing across acute care settings including ICU (see Figure 4) enabled the selection of three survey instruments for further psychometric assessment.

Figure 4. Survey instrument frequency of use

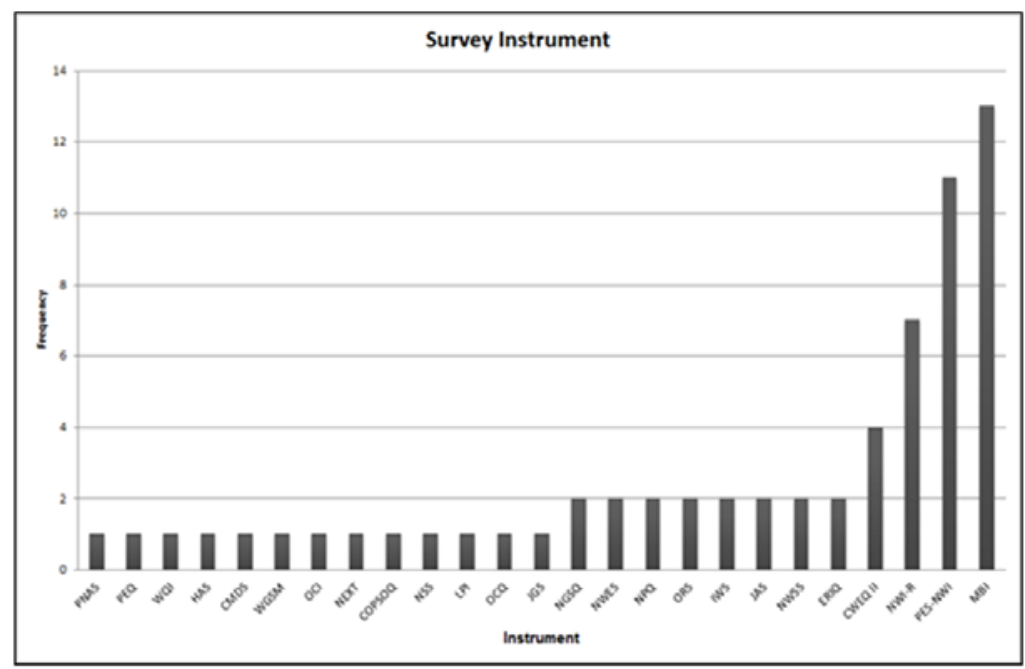

The Nurse Work Index-Revised (NWI-R) ${ }^{[94]}$, Practice Environment Scale-Nurse Work Index (PES-NWI) ${ }^{[95]}$ and Maslach's Burnout Inventory (MBI) ${ }^{[96]}$ demonstrated highest congruence and repeated testing warranting further psychometric validation.

Critical appraisal of the psychometric properties and predictive validity, of nurse survey instruments, is well establish$\mathrm{ed}^{[97-99]}$. Each survey instrument selected has undergone this process in a broad range of acute healthcare environments internationally including Australia ${ }^{[100]}$, Brazil ${ }^{[42]}$, China ${ }^{[101]}$, Japan ${ }^{[102]}$, Spain ${ }^{[103]}$, the United Kingdom ${ }^{[31]}$, United States ${ }^{[104]}$ and multi-nationally ${ }^{[105]}$. A summary of the psychometric assessment for the NWI-R, PES-NWI and MBI is provided in Table 2 .

All three survey instruments were tested repeatedly in multicenter studies involving large samples of nurses. Similarly, all instruments had been tested in acute care and ICU environments with PES-NWI being used more frequently in ICU. The content validity of the NWI-R and PES-NWI has direct relevance to the climate and culture of nurses' work environment.

The MBI focused on interpersonal and psychosocial aspects, with some relevance to organizational, factors but with a greater emphasis on individual perceptions and emotions. All three instruments have an acceptable level of reliability, with the Cronbach alpha mean composite coefficient for all studies being above 0.7 , which is recommended as the minimum threshold to establish reliability ${ }^{[106]}$.

Congruence with the nurse outcome measures was high for both the NWI-R (aligned with sixteen outcomes) and the PES-NWI (aligned with seventeen outcomes). The MBI fulfills six of the nurse outcome measures: level of participation, 
job satisfaction, emotional exhaustion (burnout), moral distress and anxiety, and depersonalization. Four outcomes captured by the MBI are not captured by the NWI-R and PES-NWI providing the justification to add subscales from the MBI to the nurse survey instrument selected.

Table 2. Survey instrument validity and congruence with nurse outcomes

\begin{tabular}{|c|c|c|c|}
\hline \multirow{2}{*}{ Quality and Validity Factors } & \multicolumn{3}{|c|}{ Survey Instrument } \\
\hline & NWI-R & PES-NWI & MBI \\
\hline Frequency & 7 & 11 & 13 \\
\hline Testing repeated & Yes (multicentre) & Yes (multicentre) & Yes (multicentre) \\
\hline Large study population & Range 155 to 2,287 & Range 67 to 98,116 & Range 155 to 98,116 \\
\hline Tested in nursing populations & Yes & Yes & Yes \\
\hline Conducted in ICU & $2 / 7$ & $4 / 11$ & $3 / 13$ \\
\hline Organizational content validity & Yes & Yes & Yes (interpersonal focus) \\
\hline Cronbach alpha: mean composite coefficient & $\alpha 85$ & $\alpha 81$ & $\alpha 82$ \\
\hline \multicolumn{4}{|l|}{ Congruence with Nurse Outcomes } \\
\hline Nurse Outcome & \multicolumn{3}{|c|}{ Measured } \\
\hline Autonomy & Yes & Yes & No \\
\hline Control over practice & Yes & Yes & No \\
\hline Empowerment & Yes & Yes & No \\
\hline Role conflict or ambiguity & Yes & Yes & No \\
\hline Nursing foundations & Yes & Yes & No \\
\hline Participation & Yes & Yes & Optional questions \\
\hline Leadership & Yes & Yes & No \\
\hline Collegiality (Doctor) & Yes & Yes & No \\
\hline Collegiality (Nursing) & Yes & Yes & No \\
\hline Resourcing and staffing & Yes & Yes & No \\
\hline Flexible scheduling & Yes & Yes & No \\
\hline Access to professional development & Yes & Yes & No \\
\hline Personal accomplishment & Yes & Yes & Yes \\
\hline Professional advancement/recognition & Yes & Yes & No \\
\hline Professional perception & Yes & Yes & No \\
\hline Satisfaction with nursing & No & Yes & No \\
\hline Job satisfaction & No & No & Yes \\
\hline Emotional exhaustion (burnout) & No & No & Yes \\
\hline Moral distress and anxiety & No & No & Yes \\
\hline Depersonalisation & No & No & Yes \\
\hline Intention to leave & Yes & Yes & Yes \\
\hline
\end{tabular}

Higher congruence with the identified nurse outcomes, demonstrated content and construct validity, an ability to discriminate positive work environment characteristics, repeated testing and strong psychometric properties supports selection of the PES-NWI as the preferred survey instrument.

The PES-NWI seeks to elicit information from staff regarding their felt experience and perceptions ${ }^{[100,107,108]}$. Factor analysis of data from magnet hospitals involving statistical testing of observed variables to determine correlation, internal consistency, reliability and validity across organizational domains, including ICU, was used to develop the PES-NWI ${ }^{[108]}$. A large number of studies and industry reports published since 2002 describe the use, modification, and scoring variations of the PES-NWI in five different countries, translated to three languages, across ten practice settings ${ }^{[101,103]}$. In a recent 
Australian study by Parker et al. (2010) ${ }^{[100]}$, the construct validity and reliability of the PES-NWI was tested in a random sample of 3,000 nurses working in private and public sectors demonstrating strong internal consistency with a Cronbach alpha of 0.948 . The study concluded that the PES-NWI is a reliable survey instrument for a range of clinical settings with ongoing refinement and testing based on large nursing populations underpinning its construct validity and reliability for the assessment of nurses work environment in acute care and ICU settings.

\subsection{Limitations}

This review provides an overview of nurse outcomes found to reflect structural factors within an organization and uses this outcome profile to select an appropriate survey instrument. Although a variety of study designs were included in the literature search, the studies included in the analysis were primarily cross sectional and therefore the ability to confer causality is significantly limited. Studies undertaken across a broad range of countries were included, however, only those studies published in English were reviewed which may limit generalization of any findings. Terminology for similar nurse outcomes varied widely requiring interpretation for classification purposes. Lastly, this literature review had a broad international perspective but does not account for variability in different health systems. These limitations may lead restrict the generalization of the findings of this review without further contextual validation.

\subsection{I mplications for nursing management}

This integrative review identifies the key constructs of a survey instrument that will assist policy makers and managers to better understand the factors contributing to a sustainable intensive care nurse workforce in the face of organizational change.

\section{Conclusion}

This literature review progressed through several stages of analysis to identify the most effective survey instrument to evaluate the working environment of nurses in ICU. The impact of structural factors on the work environment can be assessed by the nurse outcome measures captured within the PES-NWI survey instrument. The addition of the MBI is recommended to capture individual emotional responses. An instrument that incorporates both the PES-NWI and MBI subscales is most appropriate to evaluate the environment of nurses working in ICUs world-wide.

\section{Acknowledgements}

The authors would like to express their gratitude to Dr Eileen Lake for giving permission to reproduce the PES-NWI. The authors also would like to express their gratitude to Mind Garden, Inc. for permission so reproduce an extract of the MBI Human Services Survey.

\section{References}

[1] Adhikari, N., Rubenfeld, G. Worldwide demand for critical care. Current Opinion in Critical Care. 2011; 17(6): 620-5. PMid: 22067878. http://dx.doi.org/10.1097/MCC.0b013e32834cd39c

[2] Vincent, J. Critical care - where have we been and where are we going? Critical Care. 2013; 17(Suppl 1): S2. PMid: 23514264.

[3] Stone, P., Larson, E., Mooney-Kane, C., Smolowitz, J., Lin, S., Dick, A. Organizational climate and intensive care unit nurses' intention to leave. Critical Care Medicine. 2006; 34(7): 1907-12. PMid: 16625126. http://dx.doi.org/10.1097/01.CCM.0000218411.53557.29

[4] Kahn, J., Linde-Zwirble, W., Wunsch, H., Barnato, A., Iwashyna, T., Roberts, M., et al. Potential Value of Regionalized Intensive Care for Mechanically Ventilated Medical Patients. Am. J. Respir. Crit. Care Med. 2008; 177(3): 285-91. PMid: 18006884. http://dx.doi.org/10.1164/rccm.200708-12140C

[5] Nguyen, Y., Kahn, J., Angus, D. Reorganizing Adult Critical Care Delivery: The Role of Regionalization, Telemedicine, and Community Outreach. Am. J. Respir. Crit. Care Med. 2010; 181(11): 1164-9. PMid: 20224067. http://dx.doi.org/10.1164/rccm.200909-1441CP 
[6] Meadows, C., Rattenberry, W., Waldmann, C. Centralisation of specialist critical care services. JournaI of the Intensive Care Society. 2011; 12(2): 87-9.

[7] Rashid, M. Technology and the future of intensive care unit design. Critical Care Nursing Quarterly. 2011; 34(4): 332-60. PMid: 21921718. http://dx.doi.org/10.1097/CNQ.0b013e31822ba782

[8] College of Intensive Care Medicine of Australia and New Zealand 2010, Minimum standards for transport of critically ill patients, Melbourne IC-10.

[9] Valentin, A., Ferdinande, P. Recommendations on basic requirements for intensive care units: structural and organizational aspects. Intensive Care Medicine. 2011; 37(10): 1575. PMid: 21918847. http://dx.doi.org/10.1007/s00134-011-2300-7

[10] Roberts, D., Clark, H., Rock, B. Development and implementation of a regional intensive care health service model. Leadership in Health Services. 2013; 26(3): 232-6. http://dx.doi.org/10.1108/LHS-02-2013-0012

[11] Braungardt, T., Fought, S. Leading Change During an Inpatient Critical Care Unit Expansion. Journal of Nursing Administration. 2008; 38(11): 461-7. PMid: 18997550. http://dx.doi.org/10.1097/01.NNA.0000339476.73090.33

[12] Falahinia, G., Zareian, A., Oshvandi, K., Farhanchi, A., Moghimbigi, A. Comparison of intensive care units Structural Standards. Iranian Journal of Critical Care Nursing. 2013; 5(4): 222-7.

[13] Terwiesch, C., Diwas, K., Kahn, J. Working with capacity limitations: operations management in critical care. Critical Care vol. 2011; 15(4): 308. PMid: 21892976. http://dx.doi.org/10.1186/cc10217

[14] Rhodes, A., Moreno, R., Chiche, J. ICU structures and organization: putting together all the pieces of a very complex puzzle. Intensive Care Medicine. 2011; 37(10): 1569. PMid: 21918846. http://dx.doi.org/10.1007/s00134-011-2332-z

[15] Welton, J., Meyer, A., Mandelkehr, L., Fakhry, S., Jarr, S. Outcomes of and Resource Consumption by High-Cost Patients in the Intensive Care Unit. American Journal of Critical Care. 2002; 11: 467-73. PMid: 12233972.

[16] Moneke, N., Umeh, O. Factors Influencing Critical Care Nurses Perception of Their Overall Job Satisfaction: An Empirical Study. The Journal of Nursing Administration. 2013; 43(4): 201-7. PMid: 23528685. http://dx.doi.org/10.1097/NNA.0b013e31828958af

[17] Zayan, N., Reizian, A., Hamouda, G. Relationship between Organizational Climate and Nurse Outcomes. Journal of American Science. 2013; 2(9): 184-92.

[18] Hinno, S., Partanen, P., Vehviläinen, J. The professional nursing practice environment and nurse-reported job outcomes in two European countries: a survey of nurses in Finland and the Netherlands. Scandinavian Journal of Caring Sciences. 2012; 26(1): 133-43. PMid: 22032723. http://dx.doi.org/10.1111/j.1471-6712.2011.00920.x

[19] Twigg, D., McCullough, K. Nurse retention: A review of strategies to create and enhance positive practice environments in clinical settings. International Journal of Nursing Studies. 2014; 51(1): 85-92. PMid: 23809644. http://dx.doi.org/10.1016/j.ijnurstu.2013.05.015

[20] Garland, A. Figuring out what works: a need for more and better studies on the relationship between ICU organization and outcomes. Critical Care (London, England). 2010; 14(1): 108. PMid: 20156315. http://dx.doi.org/10.1186/cc8843

[21] Luzinski, C., Lundmark, V. State of the Science Related to Nurse Work Environments, Safe Practices, and Organizational Outcomes. Journal of Nursing Administration. 2012; 42(10): S1-S2. PMid: 22976888. http://dx.doi.org/10.1097/01.NNA.0000420388.10661.75

[22] Schloffman, D., Hage, A. Listen to your nurses! Improving work environments. Nursing Management. 2012; 43(9): 50-3. PMid: 22929076. http://dx.doi.org/10.1097/01.NUMA.0000418778.76679.3d

[23] Cooper, H. Scientific Guidelines for Conducting Integrative Research Reviews. Review of Educational Research. 1982; 52(2): 291-302. http://dx.doi.org/10.3102/00346543052002291

[24] Dixon-Woods, M., Agarwal, S., Young, B., Jones, D., Sutton, A. Integrative approaches to qualitative and quantitative evidence. Health Development Agency. London. 2004.

[25] Whittemore, R., Knafl, K. The integrative review: updated methodology. J Adv Nurs. 2005; 52(5): 546-53. PMid: 16268861. http://dx.doi.org/10.1111/j.1365-2648.2005.03621.x

[26] Manojlovich, M. Linking the practice environment to nurses' job satisfaction through nurse-physician communication. Journal of Nursing Scholarship. 2005; 37(4):367-73. PMid: 16396411. http://dx.doi.org/10.1111/j.1547-5069.2005.00063.x

[27] Haut, E., Sicoutris, C., Meredith, D., Sonnad, S., Reilly, P., Schwab, C., et al. Improved nurse job satisfaction and job retention with the transition from a "mandatory consultation" model to a "semiclosed" surgical intensive care unit: A 1-year prospective evaluation. Critical Care Medicine. 2006; 34(2): 387-95. PMid: 16424719. http://dx.doi.org/10.1097/01.CCM.0000198104.28666.C0

[28] Spetz, J., Herrera, C. Changes in nurse satisfaction in California, 2004 to 2008. Journal of Nursing Management. 2010; 18(5): 564-72. PMid: 20636505. http://dx.doi.org/10.1111/j.1365-2834.2010.01117.x

[29] Manojlovich, M., Antonakos, C., Ronis, D. Intensive Care Units, Communication Between Nurses and Physicians, and Patients' Outcomes. American Journal of Critical Care. 2009; 18(1): 21-30. PMid: 19116401. http://dx.doi.org/10.4037/ajcc2009353 
[30] Cho, S., Mark, B., Yun, S., June, K. Differences in intensive care unit work environments among and within hospitals using subscales and a composite measure of the Revised Nursing Work Index. Journal of Advanced Nursing. 2011. PMid: 21645043. http://dx.doi.org/10.1111/j.1365-2648.2011.05713.x

[31] Duddle, M., Boughton, M. Development and psychometric testing of the Nursing Workplace Relational Environment Scale (NWRES). J Clin Nurs. 2009; 18(6): 902-9. PMid: 19239669.

[32] Li, Y., Lake, E., Sales, A., Sharp, N., Greiner, G., Lowy, E., et al. Measuring nurses' practice environments with the revised nursing work index: Evidence from registered nurses in the veterans' health administration. Research in Nursing \& Health. 2007; 30(1): 31-44. PMid: 17243106. http://dx.doi.org/10.1002/nur.20172

[33] Liou, S., Cheng, C. Using the Practice Environment Scale of the Nursing Work Index on Asian nurses. Nursing Research. 2009; 58(3): 218-25. PMid: 19448526. http://dx.doi.org/10.1097/NNR.0b013e3181a308cd

[34] Slater, P., O'Halloran, P., Connolly, D., McCormack, B. Testing of the Factor Structure of the Nursing Work Index-Revised. Worldviews on Evidence-Based Nursing. 2010; 7(3): 123-34. PMid: 19656353. http://dx.doi.org/10.1111/j.1741-6787.2009.00158.x

[35] Miller, J. Critical Review of Quantitative Research. British Columbia. Viewed March 2013. Available from: http://www.northernhealth.ca/YourHealth/ResearchandEvaluation/ResearchEvaluationEIPResources.aspx.

[36] Von Elm, E., Altman, D., Egger, M., Pocock, S., Gøtzsche, P., Vandenbroucke, J. The Strengthening the Reporting of Observational Studies in Epidemiology (STROBE) Statement: Guidelines for reporting observational studies. Preventive Medicine. 2007; 45(4): 247-51. PMid: 17950122. http://dx.doi.org/10.1016/j.ypmed.2007.08.012

[37] Evans, D. Hierarchy of evidence: a framework for ranking evidence evaluating healthcare interventions. Journal of Clinical Nursing. 2003; 12(1):77-84. PMid: 12519253. http://dx.doi.org/10.1046/j.1365-2702.2003.00662.x

[38] Beck, C. The effects of postpartum depression on maternal-infant interaction: A meta-analysis. Nursing Research. 1995; 44(5): 298-304. PMid: 7567486. http://dx.doi.org/10.1097/00006199-199509000-00007

[39] Li, Y., Jones, C. A literature review of nursing turnover costs. Journal of Nursing Management. 2012; 21(3): 405-18. PMid: 23406301. http://dx.doi.org/10.1111/j.1365-2834.2012.01411.x

[40] Inglis, S., Clark, R., McAlister, F., Ball, J., Lewinter, C., Cullington, D., et al. Structured telephone support or telemonitoring programmes for patients with chronic heart failure. Cochrane database of systematic reviews. 2010; no. 8: 1-142.

[41] Aitken, L., Burmeister, E., Clayton, S., Dalais, C., Gardner, G. The impact of Nursing Rounds on the practice environment and nurse satisfaction in intensive care: Pre-test post-test comparative study. International Journal of Nursing Studies. $2010 ; 15$.

[42] Gasparino, R., De Brito Guirardello, E., Aiken, L. Validation of the Brazilian version of the Nursing Work Index-Revised (B-NWI-R). Journal of Clinical Nursing. 2011; 3494-3501. PMid: 21749511. http://dx.doi.org/10.1111/j.1365-2702.2011.03776.x

[43] Myhren, H., Ekeberg, O., Stokland, O. Job Satisfaction and Burnout among Intensive Care Unit Nurses and Physicians. Critical Care Research and Practice. 2013; 1-6. http://dx.doi.org/10.1155/2013/786176

[44] Aiken, L., Clarke, S., Sloane, E., Lake, E., Cheney, T. Effects of Hospital Care Environment on Patient Mortality and Nurse Outcomes. Journal of Nurse Administration. 2008; 38(5): 223-9. PMid: 18469615.

http://dx.doi.org/10.1097/01.NNA.0000312773.42352.d7

[45] Aiken, L., Sloane, D., Clarke, S., Poghosyan, L., Cho, E., You, L., et al. Importance of work environments on hospital outcomes in nine countries. International Journal for Quality in Health Care. 2011; 23(4): 357-64. PMid: 21561979. http://dx.doi.org/10.1093/intqhc/mzr022

[46] Karanikola, M., Papathanassoglou, E., Kalafati, M., Stathopoulou, H., Mpouzika, M., Goutsikas, C. Exploration of the Association between Professional Interactions and Emotional Distress of Intensive Care Unit Nursing Personnel. Dimensions of Critical Care Nursing. 2012; 31(1) 37-45. PMid: 22156812. http://dx.doi.org/10.1097/DCC.0b013e31823a55b8

[47] Van Bogaert, P., Clarke, S., Roelant, E., Meulemans, H., Van de Heyning, P. Impacts of unit-level nurse practice environment and burnout on nurse-reported outcomes: a multilevel modelling approach. Journal of Clinical Nursing. 2010; 19(11-12): 1664-74. PMid: 20579204. http://dx.doi.org/10.1111/j.1365-2702.2009.03128.x

[48] Papathanassoglou, E., Karanikola, M., Kalafati, M., Giannakopoulou, M., Lemonidou, C., Albarran, J. Professional autonomy, collaboration with physicians, and moral distress among European intensive care nurses. American Journal of Critical Care. 2012; 21(2): 41-52. PMid: 22381995. http://dx.doi.org/10.4037/ajcc2012205

[49] Duffield, C., Diers, D., O'Brien-Pallas, L., Aisbett, C., Roche, M., King, M., et al. Nursing staffing, nursing workload, the work environment and patient outcomes. Applied Nursing Research. 2011; 24(4): 244-55. PMid: 20974086. http://dx.doi.org/10.1016/j.apnr.2009.12.004

[50] Lai, H., Lin, Y., Chang, H., Wang, S., Liu, Y., Lee, H., et al. Intensive care unit staff nurses: predicting factors for career decisions. Journal of Clinical Nursing. 2008; 17(14): 1886-96. PMid: 18592616. http://dx.doi.org/10.1111/j.1365-2702.2007.02180.x 
[51] Meeusen, V., Van Dam, K., Brown-Mahoney, C., Van Zundert, A., Knape, H. Understanding nurse anesthetists' intention to leave their job: how burnout and job satisfaction mediate the impact of personality and workplace characteristics. Health Care Manage Review. 2011; 36(2): 155-63. PMid: 21317664. http://dx.doi.org/10.1097/HMR.0b013e3181fb0f41

[52] Klopper, H., Coetzee, S., Pretorius, R., Bester, P. Practice environment, job satisfaction and burnout of critical care nurses in South Africa. Journal of Nursing Management. 2012; 20(5): 685-95. PMid: 22823225. http://dx.doi.org/10.1111/j.1365-2834.2011.01350.x

[53] Iliopoulou, K., While, A. Professional autonomy and job satisfaction: survey of critical care nurses in mainland Greece. Journal of Advanced Nursing. 2010; 66(11): 2520-31. PMid: 20735503. http://dx.doi.org/10.1111/j.1365-2648.2010.05424.x

[54] Cho, S., June, K., Kim, Y., Cho, Y., Yoo, C., Yun, S., et al. Nurse staffing, quality of nursing care and nurse job outcomes in intensive care units. Journal of Clinical Nursing. 2009; 18(12): 1729-37. PMid: 19646118. http://dx.doi.org/10.1111/j.1365-2702.2008.02721.x

[55] Coetzee, S., Klopper, H., Ellis, S., Aiken, L. A tale of two systems - Nurses practice environment, wellbeing, perceived quality of care and patient safety in private and public hospitals in South Africa: A questionnaire survey. International Journal of Nursing Studies. 2013; 50(2): 162-73. PMid: 23218020. http://dx.doi.org/10.1016/j.ijnurstu.2012.11.002

[56] Van Bogaert, P., Olaf, T., Weeks, S., Van Heusden, D., Wouters, K., Franck, E. Nursing Unit Teams Matter: Impact of Unit-level Nurse Practice Environment, Nurse Work Characteristics, and Burnout on Nurse Reported Job Outcomes, and Quality of Care, and Patient Adverse Events. International Journal of Nursing Studies. 2013.

[57] Klopper, H., Aiken, L., Coetzee, S. Hospital Work Environments, Nurse Burnout and Job Dissatisfaction: A View in the Mirror of International Evidence. Paper presented to the Sigma Theta Tau International Biennial Convention (41st). Texas USA. 2012. http://hdl.handle.net/10755/201847.

[58] Aiken, L., Patrician, P. Measuring Organizational Traits of Hospitals: The Revised Nursing Work Index. Nursing Research. 2000; 49(3): 146-53. PMid: 10882319. http://dx.doi.org/10.1097/00006199-200005000-00006

[59] Neff, D., Cimiotti, J., Heusinger, A., Aiken, L. Nurse Reports from the Frontlines: Analysis of a Statewide Nurse Survey. Nursing Forum. 2011; 46(1): 4-10. PMid: 21306390. http://dx.doi.org/10.1111/j.1744-6198.2010.00201.x

[60] Roche, M., Duffield, C. A Comparison of the Nursing Practice Environment in Mental Health and Medical-Surgical Settings. Journal of Nursing Scholarship. 2010; 42(2): 195-206. PMid: 20618603. http://dx.doi.org/10.1111/j.1547-5069.2010.01348.x

[61] Van Bogaert, P., Clarke, S., Vermeyen, K., Meulemans, H., Van de Heyning, P. Practice environments and their associations with nurse-reported outcomes in Belgian hospitals: Development and preliminary validation of a Dutch adaptation of the Revised Nursing Work Index. International Journal of Nursing Studies. 2009; 46(1): 55-65. PMid: 18789437. http://dx.doi.org/10.1016/j.ijnurstu.2008.07.009

[62] Manojlovich, M., Laschinger, H. Application of the Nursing Worklife Model to the ICU Setting. Critical Care Nursing Clinics of North America. 2008; 20(4): 481-7. PMid: 19007714. http://dx.doi.org/10.1016/j.ccell.2008.08.004

[63] Duffield, C., Roche, M., Blay, N., Stasa, H. Nursing unit managers, staff retention and the work environment. Journal of Clinical Nursing. 2010; 20(1-2): 23-33. PMid: 21158986. http://dx.doi.org/10.1111/j.1365-2702.2010.03478.x

[64] Faulkner, J., Laschinger, H. The effects of structural and psychological empowerment on perceived respect in acute care nurses. J Nurs Manag. 2008; 16(2): 214-21. PMid: 18269553. http://dx.doi.org/10.1111/j.1365-2834.2007.00781.x

[65] Minvielle, M., Aegerter, P., Dervaux, B., Boumendil, A., Retbif, A., Jars-Guincestre, M., et al. Culture, organization, and management in intensive care: construction and validation of a multidimensional questionnaire. Journal of Critical Care. 2005; 20(2): 126-38. PMid: 16139153. http://dx.doi.org/10.1016/j.jcrc.2004.12.003

[66] Middleton, S., Griffiths, R., Fernandez, R., Smith, B. Nursing practice environment: How does one Australian hospital compare with magnet hospitals? International Journal of Nursing Practice. 2008; 14(5): 366-72. PMid: 18808537. http://dx.doi.org/10.1111/j.1440-172X.2008.00708.x

[67] Purdy, N., Spence Laschinger, H., Finegan, J., Kerr, M., Olivera, F. Effects of work environments on nurse and patient outcomes. Journal of Nursing Management. 2010; 18(8): 901-13. PMid: 21073564. http://dx.doi.org/10.1111/j.1365-2834.2010.01172.x

[68] Cai, C., Zhou, Z. Structural empowerment, job satisfaction, and turnover intention of Chinese clinical nurses. Nurs Health Sci. 2009; 11(4): 397-403. PMid: 19909448. http://dx.doi.org/10.1111/j.1442-2018.2009.00470.x

[69] Stordeur, S., D'Hoore, W. Organizational configuration of hospitals succeeding in attracting and retaining nurses. Journal of Advanced Nursing. 2007; 57(1): 45-58. PMid: 17184373. http://dx.doi.org/10.1111/j.1365-2648.2006.04095.x

[70] American Association of Critical-Care Nurses. AACN standards for establishing and sustaining healthy work environments: a journey to excellence. American Journal of Critical Care. 2005; 14(3): 187-97. PMid: 15840893.

[71] International Council of Nurses. Management of nursing and health care services: position statement, Wiley, Geneva, Switzerland. 2010. 
[72] Ritter, D. The relationship between healthy work environments and retention of nurses in a hospital setting. Journal of Nursing Management. 2011; 19(1): 27-32. PMid: 21223402. http://dx.doi.org/10.1111/j.1365-2834.2010.01183.x

[73] Callicutt, D., Norman, K., Smith, L., Nichols, A., Kring, D. Building an Engaged and Certified Nursing Workforce. Nursing Clinics of North America. 2011; 46(1): 81-7. PMid: 21320663. http://dx.doi.org/10.1016/j.cnur.2010.10.004

[74] Hickey, P., Gauvreau, K., Connor, J., Sporing, E., Jenkins, K. The Relationship of Nurse Staffing, Skill Mix, and Magnet Recognition to Institutional Volume and Mortality for Congenital Heart Surgery. The Journal of Nursing Administration. 2010; 40(5): 226-32. PMid: 20431457. http://dx.doi.org/10.1097/NNA.0b013e3181da3f71

[75] Soh, K., Soh, K., Davidson, P. The role of culture in quality improvement in the intensive care unit: A literature review. Journal of Hospital Administration. 2013; 2(2): 97-104. http://dx.doi.org/10.5430/jha.v2n2p97

[76] Upenieks, V. The Interrelationship of Organizational Characteristics of Magnet Hospitals, Nursing Leadership, and Nursing Job Satisfaction. Health Care Manager. April/May/June, 2003; 22(2): 83-98.

[77] Abualrub, R., Alghamdi, M. The impact of leadership styles on nurses' satisfaction and intention to stay among Saudi nurses. Journal of Nursing Management. 2012; 20(5): 668-78. PMid: 22823223. http://dx.doi.org/10.1111/j.1365-2834.2011.01320.x

[78] O'Brien-Pallas, L., Catling-Paull, C., Duffield, C., Roche, M. The Implications of Staff 'Churn' for Nurse Managers, Staff, And Patients. Nursing Economics. 2009; 27(2): 103-10. PMid: 19492774.

[79] Shirey, M. Authentic Leadership, Organizational Culture, and Healthy Work Environments. Critical Care Nursing Quarterly. 2009; 32(3): 189-98. PMid: 19542970. http://dx.doi.org/10.1097/CNQ.0b013e3181ab91db

[80] Lake, E. The Nursing Practice Environment. Medical Care Research and Review. 2007; 64(2): 104S-22S. PMid: 17406014. http://dx.doi.org/10.1177/1077558707299253

[81] Stockwell, D., Slonim, A. Quality and safety in the intensive care unit. Journal of Intensive Care Medicine (Sage Publications Inc.). 2006; 21(4): 199-210.

[82] Lin, F., Chaboyer, W., Wallis, M. A literature review of organizational, individual and teamwork factors contributing to the ICU discharge process. Australian critical care: official journal of the Confederation of Australian Critical Care Nurses. 2009; 22(1): 29-43. PMid: 19138531. http://dx.doi.org/10.1016/j.aucc.2008.11.001

[83] Pacheco, E., Campos, I., Seixas, J., Conejo, S., Vieira, H., Mazutti, S., et al. Daily multidisciplinary rounds reduce ICU length of stay. Critical Care. 2011; 15(0): 1-31.

[84] Parker, V., Giles, M., Higgins, I. Challenges confronting clinicians in acute care. J Nurs Manag. 2009; 17(6): 667-78. PMid: 19694911. http://dx.doi.org/10.1111/j.1365-2834.2009.01009.x

[85] Gershon, R., Stone, P., Bakken, S., Larson, E. Measurement of Organizational Culture and Climate in Healthcare. Journal of Nursing Administration. 2004; 34(1): 33-40. PMid: 14737033. http://dx.doi.org/10.1097/00005110-200401000-00008

[86] Hayes, B., Bonner, A., Pryor, J. Factors contributing to nurse job satisfaction in the acute hospital setting: a review of recent literature. Journal of Nursing Management. 2010; 18(7): 804-14. PMid: 20946216. http://dx.doi.org/10.1111/j.1365-2834.2010.01131.x

[87] O'Brien-Pallas, L., Murphy, G., Shamian, J., Li X, Hayes, L. Impact and determinants of nurse turnover: a pan-Canadian study. Journal of Nurse Management. 2010; 18(8): 1073-86. PMid: 21073578. http://dx.doi.org/10.1111/j.1365-2834.2010.01167.x

[88] Webster, J., Flint, A., Courtney, M. A new practice environment measure based on the reality and experiences of nurses working lives. Journal of Nursing Management. 2009; 17(1): 38-48. PMid: 19166521. http://dx.doi.org/10.1111/j.1365-2834.2008.00908.x

[89] Bartram, T., Casimir, G., Djurkovic, N., Leggat, S., Stanton, P. Do perceived high performance work systems influence the relationship between emotional labour, burnout and intention to leave? A study of Australian nurses. Journal of Advanced Nursing. 2012; 68(7): 1567-78. PMid: 22384981. http://dx.doi.org/10.1111/j.1365-2648.2012.05968.x

[90] Lederer, W., Kinzl, J.F., Traweger, C., Dosch, J., Sumann, G. Fully developed burnout and burnout risk in intensive care personnel at a university hospital. Anaesthesia and Intensive Care. 2008; 36(2): 208-13. PMid: 18361012.

[91] Mullarkey, M., Duffy, A., Timmins, F. Trust between nursing management and staff in critical care: a literature review. Nursing in Critical Care. 2011; 16(2): 85-91. PMid: 21299761. http://dx.doi.org/10.1111/j.1478-5153.2010.00404.x

[92] Groff-Paris, L., Terhaar, M. Using Maslow's pyramid and the national database of nursing quality indicators(R) to attain a healthier work environment. Online Journal of Issues in Nursing. 2011; 16(1): 1-13.

[93] Spence Laschinger, H., Almost, J., Tuer-Hodes, D. Workplace empowerment and magnet hospital characteristics: making the link. J Nurs Adm. 2003; 33(7-8): 410-22. http://dx.doi.org/10.1097/00005110-200307000-00011

[94] Lake, E. Development of the practice environment scale of the nursing work index. Research in Nursing \& Health. 2002; 25(3): 176-88. PMid: 12015780. http://dx.doi.org/10.1002/nur.10032

[95] Maslach, C., Jackson, S., Leiter, M. Maslach Burnout Inventory Manual, 3 edn, Consulting Psychologists Press, Mountain View, California. 1996. 
[96] Geurts, S., Taris, T., Kompier, M., Dikkers, J., Van Hooff, M., Kinnunen, U. Work-home interaction from a work psychological perspective: Development and validation of a new questionnaire, the SWING. Work \& Stress. 2005; 19(4): 319-39. http://dx.doi.org/10.1080/02678370500410208

[97] Streiner, D.L., Norman, G.R. Health measurement scales: a practical guide to their development and use. Oxford university press. 2008. http://dx.doi.org/10.1093/acprof:oso/9780199231881.001.0001

[98] Warshawsky, N.E., Havens, D.S. Global Use of the Practice Environment Scale of the Nursing Work Index. Nursing Research. 2011; 60(1): 17-31. PMid: 21127450. http://dx.doi.org/10.1097/NNR.0b013e3181ffa79c

[99] Parker, D., Tuckett, A., Eley, R., Hegney, D. Construct validity and reliability of the Practice Environment Scale of the Nursing Work Index for Queensland nurses. International Journal of Nursing Practice. 2010; 16(4): 352-8. PMid: 20649666. http://dx.doi.org/10.1111/j.1440-172X.2010.01851.x

[100] Zhao, P., Chen, F., Hui Jia, X., Lv, H., Cheng, P., Zhang, L. The validation and application of the Chinese version of perceived nursing work environment scale. Journal of Clinical Nursing. 2013; 22(13): 1827-32. PMid: 23647481. http://dx.doi.org/10.1111/jocn.12229

[101] Ogata, Y., Nagano, M., Fukuda, T., Hashimoto, M. Job retention and nursing practice environment of hospital nurses in Japan applying the Japanese version of the Practice Environment Scale of the Nursing Work Index (PES-NWI). Nihon Koshu Eisei Zasshi. 2011; 58(6): 409-19. PMid: 21970075.

[102] Fuentelsaz-Gallego, C., Moreno-Casbas, M., González-María, E. Validation of the Spanish version of the questionnaire Practice Environment Scale of the Nursing Work Index. International Journal of Nursing Studies. 2012; 50(2): 274-80. PMid: 22944284. http://dx.doi.org/10.1016/j.ijnurstu.2012.08.001

[103] Weston, M. Validity of Instruments for Measuring Autonomy and Control Over Nursing Practice. Journal of Nursing Scholarship. 2009; 41(1): 87-94. PMid: 19335682. http://dx.doi.org/10.1111/j.1547-5069.2009.01255.x

[104] Poghosyan, L., Aiken, L., Sloane, D. Factor structure of the Maslach burnout inventory: An analysis of data from large scale cross-sectional surveys of nurses from eight countries. International Journal of Nursing Studies. 2009; 46(7): 894-902. PMid: 19362309. http://dx.doi.org/10.1016/j.ijnurstu.2009.03.004

[105] Bonneterre, V., Liaudy, S., Chatellier, G., Lang, T., De Gaudemaris, R. Reliability, Validity, and Health Issues Arising From Questionnaires Used to Measure Psychosocial and Organizational Work Factors (POWFs) Among Hospital Nurses: A Critical Review. Journal of Nursing Measurement. 2008; 16(3): 207-30. PMid: 19886473. http://dx.doi.org/10.1891/1061-3749.16.3.207

[106] Duffield, C., Roche, M., O'Brien-Pallas, L., Catling-Paull, C., King, M. Staff satisfaction and retention and the role of the Nursing Unit Manager. Collegian (Royal College of Nursing, Australia). 2009; 16(1): 11-7. http://dx.doi.org/10.1016/j.colegn.2008.12.004

[107] Lake, E., McHugh, M. Revision of the Practice Environment Scale of the Nursing Work Index. 25th Academy Health Meeting ed. University of Pennsylvania. Washington. 2008.

[108] Harris, M. Factor Structure of the Practice Environment Scale-Nursing Work Index. Paper presented to the Midwest Nursing Research Society Conference (31st), University of Nebraska. 2007 March 23-26th. 